\title{
Chaos in three-dimensional higher spin gravity
}

\section{Prithvi Narayan ${ }^{a}$ and Junggi Yoon ${ }^{b}$}

${ }^{a}$ Department of Physics, Indian Institute of Technology Palakkad, Kozhippara P.O., Palakkad 678557, India

${ }^{b}$ School of Physics, Korea Institute for Advanced Study, 85 Hoegiro Dongdaemun-gu, Seoul 02455, Republic of Korea

E-mail: prithvi.narayan@gmail.com, junggiyoon@kias.re.kr

Abstract: We discuss $\operatorname{SL}(N, \mathbb{C})$ Chern-Simons higher spin gauge theories in Euclidean $\mathrm{AdS}_{3}$. With appropriate boundary term, we derive the higher spin generalization of Schwarzian on-shell action. We investigate gravitationally dressed bi-locals, and we study the soft higher spin mode expansion to obtain soft mode eigenfunctions. We also derive the spin- $s$ eigenfunction from Ward identity of $\mathcal{W}$-algebra and a recursion relation. Using the on-shell action, we evaluate the contributions of the soft higher spin modes to the out-of-time-order correlators, and the corresponding Lyapunov exponent of $\operatorname{SL}(N)$ higher spin gravity is found to be $\frac{2 \pi}{\beta}(N-1)$.

KeYwords: AdS-CFT Correspondence, Black Holes, Conformal and W Symmetry, Higher Spin Gravity

ARXIV EPRINT: 1903.08761 


\section{Contents}

1 Introduction 1

$2 \quad \mathcal{W}$-Schwarzian action in higher spin gravity 3

2.1 Review: Chern-Simons higher spin gravity for Euclidean $\mathrm{AdS}_{3} \quad 3$

2.2 Asymptotic AdS solution and on-shell action 6

3 Gravitational dressing and soft higher spin expansion $\quad 10$

3.1 Matter master field in Vasiliev equation 11

$\begin{array}{ll}3.2 & \text { Wilson line } \\ 3.34\end{array}$

3.3 Null relation and Ward identity of $\mathcal{W}$-algebra 17

$\begin{array}{ll}3.4 & \text { Recursion relation of soft mode eigenfunction } 20\end{array}$

4 OTOC and Lyapunov exponent $\quad 22$

$\begin{array}{lll}5 & \text { Conclusion } & 27\end{array}$

$\begin{array}{lr}\text { A Conventions } & 29\end{array}$

B Conjecture on null relation and $\mathcal{W}$ transformation 30

C Recursion relation, orthogonality and normalization 32

D Lyapunov exponent from spin- $s$ soft mode $\quad 34$

$\begin{array}{ll}\text { E OTOC in } 2 D \text { higher spin gravity } & 36\end{array}$

$\begin{array}{lll}\text { F Higher spin Schwarzian and connections to Toda theory } & 39\end{array}$

\section{Introduction}

Recently, chaotic systems have been extensively investigated in the context of AdS/CFT correspondence. One way to characterize the chaos is the butterfly effect measuring the sensitivity to the initial condition of a system. In classical system, the butterfly effect is measured by $\frac{\delta q(t)}{\delta q(0)}$ which grows exponentially in time in the chaotic system. In quantum theories, the out-of-time-ordered correlator (OTOC) has emerged as a new way to quantify the quantum chaos [1-4]. The exponential growth rate $\lambda_{L}$ of OTOCs, so-called Lyapunov exponent, is one of the important measurement of chaos, and it can be considered as a universal characteristic of chaotic system. Unlike classical system, it was shown [5] that the Lyapunov exponent of a reasonable physical system ${ }^{1}$ is bounded by $\frac{2 \pi}{\beta}$ where $\beta$ is the

\footnotetext{
${ }^{1}$ e.g., a system with large degrees of freedom (e.g., Large $N$ ), unitarity, causality and analyticity of correlation functions.
} 
inverse temperature of the system. i.e.,

$$
\lambda_{L} \leqq \frac{2 \pi}{\beta}
$$

The bound on chaos provides a natural concept of maximally chaotic system. Even before this result was known, it was already anticipated that black holes are maximally chaotic (the fastest scrambler) [6], and it was indeed shown that the black hole has maximal Lyapunov exponent by various techniques including geodesic distance [1], elastic eikonal approximation [3, 7], vacuum conformal block [8-10] and the on-shell action [11-16].

In the context of AdS/CFT correspondence, field theory dual to black hole in Einstein gravity should also be maximally chaotic. Although many interesting models are believed to be maximally chaotic, it is, in general, difficult to evaluate the OTOCs explicitly. Recently, quantum mechanical models such as Sachdev-Ye-Kitaev (SYK) models [17-23] and SYKlike tensor models [24-28] were shown to be maximally chaotic, and they have provide fruitful playground to investigate the black hole physics holographically. See reviews $[29,30]$ and recent interesting development in this direction [31-60].

The low energy dynamics of these maximally chaotic quantum mechanical models is universally governed by Schwarzian action, which plays an important role in the saturation of the chaos bound [20]. This Schwarzian action has also been observed as an on-shell action of the dual 2D gravity $[11,61-64]$ as well as the dimensional reduction of higher dimensional black hole $[15,65,66]$. Furthermore, it was found as an on-shell action of 3D gravity: metric-like formulation [16] and frame-like Chern-Simons formulation [67].

In Chern-Simons gravity $[68,69]$ with boundary, it is natural to have the Schwarzian on-shell action. The variational principle of Chern-Simons theory with boundary requires an appropriate boundary term. When this boundary term breaks the gauge symmetry on the boundary which corresponds, roughly speaking, to the conformal symmetry on the boundary, one gets anomaly of the conformal symmetry as an on-shell action.

The Chern-Simons gravity can be generalized into Chern-Simons higher spin gravity [70-72]. The higher spin gravity [73-80] in AdS and its dual CFT [81] have provided a promising laboratory to test holography [82-89]. The quantum chaos in the higher spin theories was explored by $\mathcal{W}$ vacuum blocks [10] and by Wilson line in the charge shock wave background [90]. In the Chern-Simons higher spin gravity, the corresponding higher spin on-shell action will be the anomaly of its asymptotic symmetry, so-called $\mathcal{W}$ symmetry. The form of anomaly, which is the generalization of the Schwarzian derivative, has been observed in the various literature [91-95].

In this paper, we will show how this anomaly can appear as the on-shell action ${ }^{2}$ of the higher spin gravity by choosing a appropriate boundary term and the corresponding boundary condition in [96]. For this, we consider a constant background like BTZ black hole and its smooth fluctuations which does not change the holonomy of the connection up to conjugation. This leads to the quadratic on-shell boundary action $I_{\text {on-shell }}^{(2)}$ for the smooth

\footnotetext{
${ }^{2}$ See [94] for the on-shell action for 2D higher spin BF theory.
} 
fluctuations $\eta_{n}$ and $\zeta_{n}$ corresponding to spin-2 and spin-3, respectively:

$$
\begin{aligned}
I_{\text {on-shell }}^{(2)} \sim & \frac{2 \pi^{4} i k_{c s}}{\tau^{3}} \sum_{n \geqq 2} n^{2}\left(n^{2}-1\right) \eta_{-n} \eta_{n}+\frac{\pi^{6} i k_{c s}}{3 \tau^{5}} \sum_{n \geqq 3} n^{2}\left(n^{2}-1\right)\left(n^{2}-4\right) \zeta_{-n} \zeta_{n} \\
& -(\text { anti-holomorphic) } .
\end{aligned}
$$

From the quadratic action, we could read off the propagator of the fluctuations. In addition, we study gravitationally dressed bi-locals such as (boundary-to-boundary) Wilson line or master field in the Vasiliev equation. And, we investigate the "soft" mode expansion thereof which leads to the Ward identity of $\mathcal{W}$-algebra for the bi-locals. Here, "soft" denotes a smooth infinitesimal residual gauge transformation from the constant background so that it is not involved with any large gauge transformation. Using the soft mode expansion, we will analyze the quantum chaos of the higher spin gravity based on the on-shell action.

The outline of this paper is as follows. In section 2, we review the Chern-Simons higher spin gravity with boundary, and we derive the generalized Schwarzian action as the on-shell action. In section 3, we consider master field in Vasiliev equation and Wilson line in the non-constant background, and we study the soft mode expansion thereof. In addition, based on the null relation in $\mathcal{W}_{N}$ minimal model we derive the Ward identity of $\mathcal{W}$-algebra. We also construct a recursion relation of the soft mode eigenfunction for general $s$. In section 4 , we evaluate the contribution of higher spin fields to the OTOCs to read off Lyapunov exponent of higher spin gravity. In section 5, we make concluding remarks and present the future directions. In appendix $\mathrm{B}$, we propose a conjecture on the null relation for general spin $s$ generators in $\mathcal{W}_{N}$ minimal model. In appendix $\mathrm{C}$, we provide elaborate on the recursion relations of soft mode eigenfunctions. In appendix D, we estimate the contribution of spin $s$ soft mode to Lyapunov exponent. In appendix E, we quickly apply our techniques to $2 \mathrm{D}$ higher spin BF theory to evaluate the Lyapunov exponent. In appendix F, we discuss the relation between charges in SL(3) Chern-Simons higher spin gravity and the charge in Toda field theory.

\section{$2 \mathcal{W}$-Schwarzian action in higher spin gravity}

\subsection{Review: Chern-Simons higher spin gravity for Euclidean $\mathrm{AdS}_{3}$}

We begin with the review of $\operatorname{SL}(N, \mathbb{C})$ Chern-Simons higher spin gauge gravity for Euclidean $\mathrm{AdS}_{3}[70,71,97]$. Especially, we will use $(\tau, \bar{\tau})$ formalism in [96]. Also, since we are interested in evaluation of the out-of-time-ordered correlator (OTOC) from the on-shell action via analytic continuation, we consider Euclidean $\mathrm{AdS}_{3}\left(\mathrm{EAdS}_{3}\right)$.

The three-dimensional pure higher spin gauge theories in $E A d S_{3}$ can be formulated by Chern-Simons action with complex Lie algebra $\operatorname{SL}(N, \mathbb{C})$ :

$$
I_{C S}=\frac{i k_{c s}}{4 \pi} \int_{\mathcal{M}} \operatorname{Tr}[C S(A)-C S(\bar{A})],
$$

where $k_{c s}=\frac{l}{4 G}$ is Chern-Simons level and $C S(A)$ is defined by

$$
C S(A)=A \wedge d A+\frac{2}{3} A \wedge A \wedge A .
$$


In addition, the trace $\operatorname{Tr}$ is defined in term of the trace tr over the fundamental representation by

$$
\operatorname{Tr}(M)=\frac{1}{\operatorname{tr}\left(L_{0} L_{0}\right)} \operatorname{tr}(M),
$$

where $L_{0}, L_{ \pm 1}$ are the generator of $\operatorname{sl}(2)$ subalgebra of $s l(N)$, and we will consider the principal embedding of $s l(2)$ into $s l(N)$ in this work. Furthermore, we use convention for $s l(N)$ generators such that

$$
\left(L_{n}^{(s)}\right)^{\dagger}=(-1)^{n} L_{-n}^{(s)} \quad(s=2,3, \cdots, N) .
$$

For the representation for the generators, see appendix A for $N=2,3$ cases. $^{3}$ Also, in our convention, the Chern-Simons connection $A$ is related to its conjugation $\bar{A}$ by

$$
\bar{A}=-A^{\dagger} .
$$

We use the coordinates $(r, z, \bar{z})$ given by

$$
z \equiv \phi+i \frac{t_{E}}{l}, \quad \bar{z} \equiv \phi-i \frac{t_{E}}{l} .
$$

The manifold of Chern-Simons gravity for Euclidean $\mathrm{AdS}_{3}$ is a solid torus of which the modular parameter $\tau$ of the boundary torus gives the periodicity of $z$ coordinate:

$$
z \sim z+2 \pi \sim z+\tau
$$

For BTZ black hole, the modular parameter $\tau$ is given by ${ }^{4}$

$$
\begin{aligned}
& \tau=w+\frac{i \beta}{l}=\frac{2 \pi i l\left(r_{+}-i r_{\mathrm{E}}\right)}{r_{+}^{2}-r_{-}^{2}}=\frac{2 \pi i l}{r_{+}+r_{-}}, \\
& \bar{\tau}=w-\frac{i \beta}{l}=-\frac{2 \pi i l\left(r_{+}+i r_{\mathrm{E}}\right)}{r_{+}^{2}-r_{-}^{2}}=-\frac{2 \pi i l}{r_{+}-r_{-}} .
\end{aligned}
$$

One can fix a gauge $[71,72]$ such that

$$
\begin{aligned}
& A=b^{-1}\left(d+a_{z} d z+a_{\bar{z}} d \bar{z}\right) b, \\
& \bar{A}=b\left(d+\bar{a}_{z} d z+\bar{a}_{\bar{z}} d \bar{z}\right) b^{-1},
\end{aligned}
$$

where $b(r)$ is defined by

$$
b=e^{r L_{0}}
$$

In Chern-Simons gravity theories, the asymptotic $\mathrm{AdS}_{3}$ condition with a flat boundary metric, which is analogous to the Brown-Henneaux asymptotic AdS boundary condition in the metric-like formulation, is [71, 72]

$$
A-\left.A_{\text {AdS }}\right|_{\partial \mathcal{M}} \sim \phi(1),
$$

\footnotetext{
${ }^{3}$ For $\operatorname{sl}(N)$, see [86]. Note that we use the different realization of the $s l(3)$ generators from [96].

${ }^{4}$ Note that $r_{-}-=i r_{\mathrm{E}}$ is pure imaginary in Euclidean signature so that we will distinguish them clearly.
} 
where $A_{\text {AdS }}$ is the exact $A d S_{3}$ solution [71, 72]:

$$
A_{\mathrm{AdS}}=b^{-1}\left[d+\left(L_{1}+\frac{1}{4} L_{-1}\right) d z\right] b, \quad \bar{A}_{\mathrm{AdS}}=b\left[d+\left(L_{-1}+\frac{1}{4} L_{1}\right) d \bar{z}\right] b^{-1} .
$$

The asymptotic $\mathrm{AdS}_{3}$ condition (2.12) leads to

$$
a=L_{1} d z+\cdots, \quad \bar{a}=L_{-1} d \bar{z}+\cdots .
$$

The variation of the Chern-Simons action without any boundary term ${ }^{5}$ (2.1) gives

$$
\delta I_{C S}=-\frac{i k_{c s}}{4 \pi} \int_{\partial \mathcal{M}} \operatorname{Tr}[A \wedge \delta A-\bar{A} \wedge \delta \bar{A}]
$$

Hence, we can choose a boundary condition $A_{\bar{z}}=\bar{A}_{z}=0$ for consistent variational principle. Furthermore, by using residual gauge symmetry, we can fix the highest weight gauge [71, 72]. For $N=3$, the highest weight gauge is given by

$$
\begin{aligned}
& a_{z}(z)=L_{1}-\frac{2 \pi}{k_{c s}} \mathcal{L}(z) L_{-1}+\frac{\pi}{2 k_{c s}} \mathcal{W}(z) W_{-2}=\left(\begin{array}{ccc}
0 & \sqrt{2} \frac{2 \pi}{k_{c s}} \mathcal{L}(z) & \frac{2 \pi}{k_{c s}} \mathcal{W}(z) \\
\sqrt{2} & 0 & \sqrt{2} \frac{2 \pi}{k_{c s}} \mathcal{L}(z) \\
0 & \sqrt{2} & 0
\end{array}\right), \\
& \bar{a}_{\bar{z}}(\bar{z})=L_{-1}-\frac{2 \pi}{k_{c s}} \overline{\mathcal{L}}(\bar{z}) L_{1}+\frac{\pi}{2 k_{c s}} \overline{\mathcal{W}}(\bar{z}) W_{2}=\left(\begin{array}{ccc}
0 & -\sqrt{2} & 0 \\
-\sqrt{2} \frac{2 \pi}{k_{c s}} \overline{\mathcal{L}}(\bar{z}) & 0 & -\sqrt{2} \\
-\frac{2 \pi}{k_{c s}} \mathcal{W}(\bar{z}) & -\sqrt{2} \frac{2 \pi}{k_{c s}} \overline{\mathcal{L}}(\bar{z}) & 0
\end{array}\right) .
\end{aligned}
$$

To study generic higher spin black hole, we need to consider the variation of the modular parameter $\tau$ as well as chemical potentials for higher spin charges [96-100]. We will use the $(\tau, \bar{\tau})$ formalism in [96] to incorporate the variation of the modular parameters. Although the formalism works for generic chemical potentials, for simplicity, we turn off the chemical potential for higher spin charges - if present they become a source of the spin- $s$ Schwarzian. Hence, in this work, we focus only on BTZ black hole embedded in the higher spin gravity in which the derivation of on-shell action will formally be parallel to that of $\operatorname{SL}(2, \mathbb{C})$ case $[7]$.

Recall that the modular parameter appears as the periodicity of the coordinates $(z, \bar{z})$. Hence, for the variation of the modular parameter, it is useful to fix periodicity. For this, we introduce a new coordinate $(w, \bar{w})$ defined by

$$
z=\frac{1-i \frac{\tau}{2 \pi}}{2} w+\frac{1+i \frac{\tau}{2 \pi}}{2} \bar{w}
$$

and, the coordinates $w$ and $\bar{w}$ has a fixed periodicity:

$$
w \sim w+2 \pi \sim w+2 \pi i .
$$

By fixing the periodicity of $w, \bar{w}$, the boundary metric and the boundary volume element depends on the modular parameter:

$$
\begin{aligned}
d s^{2} & =d z d \bar{z}=\left|\left(\frac{1-i \frac{\tau}{2 \pi}}{2}\right) d w+\left(\frac{1+i \frac{\tau}{2 \pi}}{2}\right) d \bar{w}\right|^{2}, \\
i d w \wedge d \bar{w} & =\frac{4 \pi d z^{2}}{\operatorname{Im}(\tau)}
\end{aligned}
$$

\footnotetext{
${ }^{5}$ We will choose appropriate boundary term soon in (2.22).
} 
where $d z^{2}=\frac{i}{2} d z \wedge d \bar{z}$. It was the key point in [96] to keep the boundary volume element $w \wedge$ $\bar{w}$ under the variation of the action. In the variation of the Chern-Simons bulk action (2.1), it is convenient to use $(w, \bar{w})$ coordinates because the differential form $d w$ and $d \bar{w}$ are not varied under the variation. Then, after varying the action, one can return to $(z, \bar{z})$ coordinates where the variation of $a_{w}, a_{\bar{w}}$ leads to the variation of the modular parameter $\tau, \bar{\tau}$ because the linear transformation from $\left(a_{w}, a_{\bar{w}}\right)$ to $\left(a_{z}, a_{\bar{z}}\right)$ depends on $\tau$ and $\bar{\tau}$ :

$$
a_{w}=\left(\frac{1-i \frac{\tau}{2 \pi}}{2}\right) a_{z}+\left(\frac{1-i \frac{\bar{\tau}}{2 \pi}}{2}\right) a_{\bar{z}}
$$

With the boundary term chosen in [96]

$$
I_{b}=-\frac{k_{c s}}{2 \pi} \int_{\partial \mathcal{M}} d^{2} z \operatorname{Tr}\left(\left(a_{z}-2 L_{1}\right) a_{\bar{z}}\right)-\frac{k_{c s}}{2 \pi} \int_{\partial \mathcal{M}} d^{2} z \operatorname{Tr}\left(\left(\bar{a}_{\bar{z}}-2 L_{-1}\right) \bar{a}_{z}\right)
$$

the variation of the total action $I_{\mathrm{tot}} \equiv I_{C S}+I_{b}$ is

$$
\begin{aligned}
\delta I_{\mathrm{tot}}=-i k_{c s} \int_{\partial \mathcal{M}} \frac{d^{2} z}{2 \pi \operatorname{Im}(\tau)} \operatorname{Tr} & {\left[\left(a_{z}-L_{1}\right) \delta\left((\bar{\tau}-\tau) a_{\bar{z}}\right)+\left(\frac{a_{z}^{2}}{2}+a_{z} a_{\bar{z}}-\frac{\bar{a}_{z}^{2}}{2}\right) \delta \tau\right.} \\
& \left.-\left(-\bar{a}_{\bar{z}}-L_{-1}\right) \delta\left((\bar{\tau}-\tau) \bar{a}_{z}\right)+\left(\frac{\bar{a}_{\bar{z}}^{2}}{2}+\bar{a}_{\bar{z}} \bar{a}_{z}-\frac{a_{\bar{z}}^{2}}{2}\right) \delta \bar{\tau}\right] .
\end{aligned}
$$

For consistent boundary condition, we impose a boundary condition

$$
a_{\bar{z}}=\bar{a}_{z}=0, \quad \delta \tau=\delta \bar{\tau}=0 .
$$

Note that since we turn off the chemical potential for higher spin charges, the variation of the total action is simpler than that of [96]. Then, using the boundary condition we obtain the on-shell action of $I_{\text {tot }}$ :

$$
I_{\mathrm{on}-\mathrm{shell}}=\frac{i k_{c s}}{2 \pi} \int \frac{d^{2} z}{\operatorname{Im}(\tau)} \operatorname{Tr}\left[\frac{\tau}{2} a_{z}^{2}-\frac{\bar{\tau}}{2} \bar{a}_{\bar{z}}^{2}\right] .
$$

Note that though [96] mainly analyzed a constant solution $a$ and $\bar{a}$ with chemical potential, they already pointed out that their formulation can be applied to the non-constant solutions.

\subsection{Asymptotic AdS solution and on-shell action}

In this section, we will work out the asymptotic AdS solution and the corresponding onshell action for the case of SL(3, $\mathbb{C})$ explicitly. Due to the boundary condition in $(2.24)$, by using residual gauge symmetry, we can fix gauge connection to be

$$
\begin{aligned}
& a_{z}(z)=L_{1}-\frac{2 \pi}{k_{c s}} \mathcal{L}(z) L_{-1}+\frac{\pi}{2 k_{c s}} \mathcal{W}(z)=\left(\begin{array}{ccc}
0 & \sqrt{2} \frac{2 \pi}{k_{c s}} \mathcal{L}(z) & \frac{2 \pi}{k_{c s}} \mathcal{W}(z) \\
\sqrt{2} & 0 & \sqrt{2} \frac{2 \pi}{k_{c s}} \mathcal{L}(z) \\
0 & \sqrt{2} & 0
\end{array}\right), \\
& \bar{a}_{\bar{z}}(\bar{z})=L_{-1}-\frac{2 \pi}{k_{c s}} \overline{\mathcal{L}}(\bar{z}) L_{1}+\frac{\pi}{2 k_{c s}} \overline{\mathcal{W}}(\bar{z})=\left(\begin{array}{ccc}
0 & -\sqrt{2} & 0 \\
-\sqrt{2} \frac{2 \pi}{k_{c s}} \overline{\mathcal{L}}(\bar{z}) & 0 & -\sqrt{2} \\
-\frac{2 \pi}{k_{c s}} \mathcal{W}(\bar{z}) & -\sqrt{2} \frac{2 \pi}{k_{c s}} \overline{\mathcal{L}}(\bar{z}) & 0
\end{array}\right) .
\end{aligned}
$$


Then, the on-shell action in (2.25) can be written as

$$
I_{\text {on-shell }}=i \int \frac{d^{2} z}{\operatorname{Im}(\tau)}[\tau \mathcal{L}(z)-\bar{\tau} \overline{\mathcal{L}}(\bar{z})]
$$

In this work, we will study non-constant gauge connection $a$ and $\bar{a}$ that are connected to a fixed constant solution $a_{\text {Втд }}$ (e.g., BTZ black hole embedded in the higher spin gravity) by smooth gauge transformation. i.e.,

$$
a=h^{-1} a_{\mathrm{BTZ}} h+h^{-1} d h,
$$

where smooth gauge transformation means that $h(z)$ is a holomorphic residual gauge transformation parameter which can be smoothly connected to identity. We are interested in the simplest constant connection $a_{\mathrm{BTz}}$ without spin-3 charge:

$$
a_{\mathrm{BTZ}}=\left(\begin{array}{ccc}
0 & \sqrt{2} \frac{2 \pi}{k_{c s}} \mathcal{L}_{0} & 0 \\
\sqrt{2} & 0 & \sqrt{2} \frac{2 \pi}{k_{c s}} \mathcal{L}_{0} \\
0 & \sqrt{2} & 0
\end{array}\right)
$$

where $\mathcal{L}_{0}$ is a constant. Under the smooth residual gauge transformation, the holonomy is not changed up to similarity transformation.

$$
\operatorname{Hol}_{\mathcal{C}}(A) \equiv \mathcal{P} \exp \left[-\int_{\mathcal{C}} A\right]=b^{-1} h^{-1} e^{-\precsim h b} .
$$

Note that a holonomy along the contractible cycle of the solid torus should be trivial, namely, it belong to the center of the $\operatorname{SL}(N)$ gauge group [97, 99, 101]. The Euclidean time circle $\mathcal{C}$ is the contractible on in the BTZ black hole, and the smoothness of its holonomy leads to

$$
\precsim=\tau a_{z}+\bar{\tau} a_{\bar{z}}=u^{-1}\left(2 \pi i L_{0}\right) u,
$$

for some matrix $u$. From this condition, one can find the relation between the constant $\mathcal{L}_{0}$, constant energy-momentum tensor of the BTZ black hole, and the modular parameter $\tau$ :

$$
\operatorname{tr}\left(\precsim^{2}\right)=\operatorname{tr}\left[\left(\begin{array}{ccc}
0 & -\frac{2 \pi \tau \mathcal{L}_{0}}{k_{c s}} \sqrt{2} & 0 \\
\sqrt{2} \tau & 0 & -\frac{2 \pi \tau \mathcal{L}_{0}}{k_{c s}} \sqrt{2} \\
0 & \sqrt{2} \tau & 0
\end{array}\right)^{2}\right]=\frac{16 \pi \tau^{2} \mathcal{L}_{0}}{k_{c s}}=-8 \pi^{2},
$$

and, we have

$$
\tau=i \pi \sqrt{\frac{k_{c s}}{2 \pi \mathcal{L}_{0}}}, \quad \bar{\tau}=-i \pi \sqrt{\frac{k_{c s}}{2 \pi \overline{\mathcal{L}}_{0}}} .
$$

Recall that we fix the modular parameter under the variation of the action. This corresponds to fixing the constant $\mathcal{L}_{0}$ and $\overline{\mathcal{L}}_{0}$. Therefore, our on-shell action captures the (fixed) BTZ black hole geometry embedded in higher spin gravity with $\tau, \bar{\tau}$ together with the smooth fluctuation thereof.

Now, we will calculate $\mathcal{L}(z)$ in the non-constant connection $a(z)$ in terms of the smooth residual gauge transformation parameter $h(z)$ which transforms the constant background 
$a_{\text {BTZ }}$ to $a(z)$. For arbitrary constant $\mathcal{L}_{0}$, this can be worked out in principle as follow. Using the Gauss decomposition of the gauge parameter $h(z)$, one can find a generic residual gauge symmetry parameter which keep the gauge condition that we chose in (2.26). However, among the generic residual gauge symmetry parameter, it is not easy to distinguish smooth one from large gauge transformation which will change the holonomy. Unlike SL(2) case where we know the correct answer for any $\mathcal{L}_{0}$, for now we do not have a guiding principle to derive the form of $\mathcal{L}(z)$ or $\mathcal{W}(z)$ for the non-zero $\mathcal{L}_{0}$, and this corresponds to find "the finite temperature $\operatorname{SL}(N)$ Schwarzian derivative". ${ }^{6}$

At least, an infinitesimal residual gauge transformation is smooth, and one can obtain $\mathcal{L}(z)$ and $\mathcal{W}(z)$ perturbatively. But, unlike $\mathrm{SL}(2)$ case, it is not easy to integrate the perturbative expression of $\mathcal{L}(z)$ and $\mathcal{W}(z)$ to obtain the full higher spin Schwarzian derivative at finite temperature, either. Nevertheless, for our purpose, it is enough to evaluate $\mathcal{L}(z)$ perturbatively because the leading contribution of boundary higher spin modes to OTOCs can be evaluated by quadratic on-shell action. Hence, let us consider an infinitesimal residual gauge transformation by

$$
h=\mathbb{I}+\epsilon \lambda^{(1)}+\frac{1}{2} \epsilon^{2}\left[\left(\lambda^{(1)}\right)^{2}+\lambda^{(2)}\right]+\cdots,
$$

where $\lambda^{(1)}, \lambda^{(2)} \in \operatorname{sl}(3)$ are parametrized by

$$
\begin{aligned}
\lambda^{(1)} & =\left(\begin{array}{ccc}
\varphi_{1}^{(1)} & e_{-1}^{(1)} & e_{-3}^{(1)} \\
e_{1}^{(1)} & -\varphi_{1}^{(1)}+\varphi_{2}^{(1)} & e_{-2}^{(1)} \\
e_{3}^{(1)} & e_{2}^{(1)} & -\varphi_{2}^{(1)}
\end{array}\right), \\
\lambda^{(2)} & =\left(\begin{array}{ccc}
\varphi_{1}^{(2)} & e_{-1}^{(2)} & e_{-3}^{(2)} \\
e_{1}^{(2)} & -\varphi_{1}^{(2)}+\varphi_{2}^{(2)} & e_{-2}^{(2)} \\
e_{3}^{(2)} & e_{2}^{(2)} & -\varphi_{2}^{(2)}
\end{array}\right) .
\end{aligned}
$$

The infinitesimal gauge transformation leads to the expansion of the on-shell action in (2.27):

$$
I_{\mathrm{on}-\text { shell }}=I_{\mathrm{on}-\mathrm{shell}}^{(0)}+\epsilon I_{\mathrm{on}-\text { shell }}^{(1)}+\epsilon^{2} I_{\mathrm{on}-\text { shell }}^{(2)}+\cdots .
$$

We demand that the gauge transformation of the constant connection $a_{\text {Bтz }}$ by $h(z)$ keeps the gauge condition in (2.26) order by order. i.e.,

$$
\begin{aligned}
a & =h^{-1} a_{\mathrm{BTZ}} h+h^{-1} \partial_{z} h, \\
& =a_{\mathrm{BTZ}}+\epsilon\left(\begin{array}{ccc}
0 & \sqrt{2} \frac{2 \pi}{k_{c s}} \mathcal{L}^{(1)} & \frac{2 \pi}{k_{c s}} \mathcal{W}^{(1)} \\
0 & 0 & \sqrt{2} \frac{2 \pi}{k_{c s}} \mathcal{L}^{(1)} \\
0 & 0 & 0
\end{array}\right)+\epsilon^{2}\left(\begin{array}{ccc}
0 & \sqrt{2} \frac{2 \pi}{k_{c s}} \mathcal{L}^{(2)} & \frac{2 \pi}{k_{c s}} \mathcal{W}^{(2)} \\
0 & 0 & \sqrt{2} \frac{2 \pi}{k_{c s}} \mathcal{L}^{(2)} \\
0 & 0 & 0
\end{array}\right)+\cdots
\end{aligned}
$$

In the first order, one can determine $\varphi_{1}^{(1)}, \varphi_{2}^{(1)}, e_{2}^{(1)}, e_{-1}^{(1)}, e_{-2}^{(1)}, e_{-3}^{(1)}$ as a linear combinations of $e_{1}^{(1)}$ and $e_{3}^{(1)}$. Also, by demanding the gauge condition in the second order, one can

\footnotetext{
${ }^{6}$ For "zero temperature Schwarzian derivative", see appendix F and [91, 92, 94, 95]. Note that [94] proposed a conjecture on the finite temperature SL(3) Schwarzian.
} 
express $\varphi_{1}^{(2)}, \varphi_{2}^{(2)}, e_{2}^{(2)}, e_{-1}^{(2)}, e_{-2}^{(2)}, e_{-3}^{(2)}$ in terms of the quadratics of $e_{1}^{(1)}$ and $e_{3}^{(1)}$ and the linear combinations of $e_{1}^{(2)}$ and $e_{3}^{(2)}$. As a result, the first order $\mathcal{L}^{(1)}$ and $\mathcal{W}^{(1)}$ gives the $\mathcal{W}_{3}$ algebra as asymptotic symmetry with identification $c=6 k_{c s}=\frac{3 l}{2 G}[71,72,102]$. By redefining ${ }^{7}$

$$
\begin{aligned}
& \epsilon e_{1}^{(1)}(z)=\eta(z)-\frac{1}{2 \sqrt{2}} \zeta^{\prime}(z), \\
& \epsilon e_{3}^{(2)}(z)=\zeta(z)
\end{aligned}
$$

one can express the second order $\mathcal{L}^{(2)}$ and $\mathcal{W}^{(2)}$ in terms of $\eta(z)$ and $\zeta(z)$ :

$$
\begin{aligned}
\epsilon^{2} \mathcal{L}^{(2)}= & \frac{k_{c s}}{16 \pi}\left(-\left(\frac{2 \pi}{\tau}\right)^{2}\left[\eta^{\prime}\right]^{2}+\left[\eta^{\prime \prime}\right]^{2}\right) \\
& +\frac{k_{c s}}{384 \pi}\left(4\left(\frac{2 \pi}{\tau}\right)^{4}\left[\zeta^{\prime}\right]^{2}-5\left(\frac{2 \pi}{\tau}\right)^{2}\left[\zeta^{\prime \prime}\right]^{2}+\left[\zeta^{\prime \prime \prime}\right]^{3}\right), \\
\epsilon^{2} \mathcal{W}^{(2)}= & \frac{k_{c s}}{24 \sqrt{2} \pi}\left(4\left(\frac{2 \pi}{\tau}\right)^{4} \eta^{\prime} \zeta^{\prime}-5\left(\frac{2 \pi}{\tau}\right)^{2} \eta^{\prime \prime} \zeta^{\prime \prime}+\eta^{\prime \prime \prime} \zeta^{\prime \prime \prime}\right) .
\end{aligned}
$$

up to total derivatives. Here, we used the relation between the modular parameter $\tau$ and $\mathcal{L}_{0}$ in (2.33) derived from the smoothness of holonomy along the contractible cycle. Expanding $\eta(z)$ and $\zeta(z)$ by

$$
\eta(z)=\sum_{n} \eta_{n} e^{-\frac{2 \pi i n z}{\tau}}, \quad \zeta(z)=\sum_{n} \zeta_{n} e^{-\frac{2 \pi i n z}{\tau}}
$$

the quadratic on-shell action $I_{\text {on-shell }}^{(2)}$ in $(2.37)$ becomes

$$
\begin{aligned}
\epsilon^{2} I_{\text {on-shell }}^{(2)}= & \frac{2 \pi^{4} i k_{c s}}{\tau^{3}} \sum_{n \geqq 2} n^{2}\left(n^{2}-1\right) \eta_{-n} \eta_{n}+\frac{\pi^{6} i k_{c s}}{3 \tau^{5}} \sum_{n \geqq 3} n^{2}\left(n^{2}-1\right)\left(n^{2}-4\right) \zeta_{-n} \zeta_{n} \\
& -(\text { anti-holomorphic }) .
\end{aligned}
$$

Note that the on-shell action for $\eta_{n}$ and $\zeta_{n}$ vanishes for $n=0, \pm 1$ and $n=0, \pm 1, \pm 2$, respectively. They are the $\operatorname{SL}(3, \mathbb{C})$ isometry of the constant connections. In the higher spin black hole, $\mathrm{SL}(3, \mathbb{C})$ isometry of AdS vacuum is supposed to be broken because of the periodicity of $\varphi$. But, because we are working in the covering space of $\varphi$, we still have $\mathrm{SL}(3, \mathbb{C})$ isometry. Now, one can read off the two point function of boundary soft modes:

$$
\begin{aligned}
\left\langle\eta_{-n} \eta_{n}\right\rangle & =\frac{\kappa_{2}}{n^{2}\left(n^{2}-1\right)} & & (|n| \geqq 2), \\
\left\langle\zeta_{-n} \zeta_{n}\right\rangle & =\frac{\kappa_{3}}{n^{2}\left(n^{2}-1\right)\left(n^{2}-4\right)} & & (|n| \geqq 3) .
\end{aligned}
$$

where the coefficient $\kappa_{2}$ and $\kappa_{3}$ are given by

$$
\kappa_{2} \equiv \frac{\tau^{3}}{2 \pi^{4} i k_{c s}}, \quad \kappa_{3} \equiv \frac{3 \tau^{5}}{\pi^{6} i k_{c s}} .
$$

\footnotetext{
${ }^{7}$ Here, we absorbed the infinitesimal parameter $\varepsilon$ into $\eta(z)$ and $\zeta(z)$.
} 


\section{Gravitational dressing and soft higher spin expansion}

One way to diagnose quantum chaos is by the out-of-time-ordered correlators of matter field or equivalently by scattering of shock waves in the dual bulk theory. However, the threedimensional pure higher spin gauge theory does not contain a matter field. This problem is more pronounced in Chern-Simons formulation, since being topological, it is not easy to couple the higher spin fields to matter which is not topological in $\mathrm{AdS}_{3}$. The construction of an action of interacting higher spin gauge theories with matter is also a challenging problem.

Although it is difficult to construct interaction with matter field, one may consider a scalar field in the probe limit. And, in a simple background geometry such as BTZ black hole embedded in higher spin gravity, the boundary-to-boundary propagator of the probe scalar field is nothing but two point function in $\mathrm{CFT}_{2}$ with suitable conformal dimension. However, the evaluation of OTOCs from the on-shell action via analytic continuation requires the $\mathcal{W}$ transformation of the two point function, at least, infinitesimally. In contrast to the conformal transformation, the $\mathcal{W}$ transformation of two point function is not well-understood due to the non-linearity of $\mathcal{W}_{N}$ algebra.

Despite of the above obstacles, we could find ways to deal with those difficulties. For the evaluation of OTOCs via analytic continuation, we first need to calculate Euclidean four point function on the boundary. For this, we consider a particular type of four point function which can be viewed as two point function of bi-local operators $\Phi\left(z_{1}, \bar{z}_{1} ; z_{2}, \bar{z}_{2}\right)$ and its leading contribution to the four point function (equivalently, two point function of two bi-locals) is the product of one-point function of each bi-locals.

$$
\left\langle\Phi_{1}\left(z_{1}, \bar{z}_{1} ; z_{2}, \bar{z}_{2}\right) \Phi_{2}\left(z_{3}, \bar{z}_{3} ; z_{4}, \bar{z}_{4}\right)\right\rangle=G_{1}\left(z_{12}, \bar{z}_{12}\right) G_{2}\left(z_{34}, \bar{z}_{34}\right)+\cdots,
$$

where $G_{i}(z, \bar{z})(i=1,2)$ is the one-point function of the bi-local operator corresponding to a boundary-to-boundary two point function:

$$
G_{i}\left(z_{12}, \bar{z}_{12}\right) \equiv\left\langle\Phi_{i}\left(z_{1}, \bar{z}_{1} ; z_{2}, \bar{z}_{2}\right)\right\rangle \quad(i=1,2)
$$

We will consider a gravitationally dressed bi-local field, namely, the bi-local field in the non-constant background. In particular, for a non-constant background which is connected to a constant background (e.g., BTZ black hole) by infinitesimal gauge transformation, one can expand the gravitationally dressed bi-local field with respect to the soft modes.

$$
\Phi^{\text {dressed }}\left(z_{1}, \bar{z}_{1} ; z_{2}, \bar{z}_{2}\right)=G\left(z_{1}, \bar{z}_{1} ; z_{2}, \bar{z}_{2}\right)+\epsilon G^{(1)}\left(z_{1}, \bar{z}_{1} ; z_{2}, \bar{z}_{2}\right)+\cdots
$$

Recall that an infinitesimal residual gauge transformation induces an infinitesimal $\mathcal{W}$ transformation on the boundary. Therefore, the soft mode expansion of the gravitationally dressed bi-local field, which is generated by an infinitesimal residual gauge transformation from constant connection to non-constant one, gives the $\mathcal{W}$ transformation of the leading term, namely, $\mathcal{W}$ transformation of the boundary two point function.

In $[103,104]$, the complete set of conformal partial wave functions has been constructed by correlation function of bi-local operator and spin- $s$ primary operator. In this spirit, one 
can also induce the soft mode eigenfunction by taking correlation function between the dressed bi-local operator and the soft higher spin mode:

$$
\left\langle\epsilon \Phi^{\text {dressed }}\left(z_{1}, \bar{z}_{1} ; z_{2}, \bar{z}_{2}\right)\right\rangle \sim G^{(1)}\left(z_{1}, \bar{z}_{1} ; z_{2}, \bar{z}_{2}\right)+\cdots .
$$

Recall that the soft mode expansion of the dressed bi-local operator defines $G^{(1)}$ i.e., $\delta_{\epsilon} \Phi^{\text {dressed }}=G^{(1)}$. Therefore, (3.4) can be viewed as the Ward identity of $\mathcal{W}_{3}$ algebra.

In the next few sections, we will discuss the form of $G^{(1)}$ using various arguments. First, we will consider the master field of matter in the Vasiliev equation in section 3.1 and a Wilson line in section 3.2 as a gravitationally dressed bi-local operator, and we will study the soft mode expansion thereof. In addition, from the null relation of primary operators in higher spin $\mathrm{AdS}_{3} / \mathrm{CFT}_{2}$ duality to find the Ward identity of $\mathcal{W}$-algebra for its two point function in section 3.3. We also discuss recursion relations of the soft mode eigenfunctions in section 3.4.

\subsection{Matter master field in Vasiliev equation}

Let us consider the $\mathcal{W}_{N}$ minimal model at semi-classical limit where 'tHooft coupling constant $\lambda$ is taken to be $-N$ in the higher spin $\mathrm{AdS}_{3} / \mathrm{CFT}_{2}[86,105,106]$. In the semiclassical limit, the gauge sector becomes $\operatorname{sl}(N) \times \operatorname{sl}(N)$ Chern-Simons gravity where the gauge connection $A$ is $\operatorname{sl}(N)$ matrix of which equation of motion is given by

$$
d A+A \wedge A=0 .
$$

In addition, the propagating scalar field can be described by master field $C$ which is a $N \times N$ matrix [86]:

$$
d C+A C-C \bar{A}=0 .
$$

Note that the physical scalar field corresponds to the trace of the master field $C$, and other components can be expressed in terms of its derivatives [86, 107]. Note that the equations of motion are invariant under the gauge transformation

$$
\begin{aligned}
\delta A & =d \xi+A \xi-\xi A, \\
\delta C & =-\xi C+C \bar{\xi} .
\end{aligned}
$$

As in the previous section, we take into account a constant background and its fluctuation connected by smooth residual gauge transformation:

$$
A_{z}=b^{-1}\left(h^{-1} a_{\mathrm{BTZ}} h+h^{-1} \partial_{z} h\right) b, \quad A_{r}=b^{-1} \partial_{r} b,
$$

where $b(r) \equiv e^{r L_{0}}$.

The solution of the equation of motion for $C$ in the constant background is easily found to be $[86]$

$$
C(r, z, \bar{z})=b^{-1}(r) e^{-a_{\mathrm{BTZ}} z} c_{0} e^{\bar{a}_{\mathrm{BTZ}} \bar{z}} b^{-1}(r),
$$

where $c_{0}$ is a constant matrix. It was proven [86] that $\operatorname{tr}(C)$ satisfies the Klein-Gordon equation for any choice of $c_{0}$. In particular, for $\mathcal{L}_{0}=\overline{\mathcal{L}}_{0}=0$, it satisfies

$$
\left[\partial_{r}^{2}+2 \partial_{r}+4 e^{-2 r} \partial \bar{\partial}-\left(N^{2}-1\right)\right] \operatorname{tr}(C)=0 .
$$


Note that with the choice of "the highest weight state" $c_{0}$

$$
\left(c_{0}\right)_{i j}=\delta_{i 1} \delta_{j 1}
$$

it was also shown that $\operatorname{tr}(C)$ gives the boundary-to-bulk propagator [86].

One can also study the master field in a non-stationary background. The solution with non-constant connection can be written as Wilson line:

$$
\begin{aligned}
& C\left(r_{1}, z_{1}, \bar{z}_{1} ; z_{2}, \bar{z}_{2}\right), \\
& \quad=\lim _{r_{2} \rightarrow \infty} e^{2 h r_{2}} b^{-1}\left(r_{1}\right) \mathcal{P} \exp \left[-\int_{z_{2}}^{z_{1}} a\right] b\left(r_{2}\right) \tilde{c}_{0} b\left(r_{2}\right) \mathcal{P} \exp \left[-\int_{\bar{z}_{1}}^{\bar{z}_{2}} \bar{a}\right] b^{-1}\left(r_{1}\right),
\end{aligned}
$$

where $\tilde{c}_{0}$ is a constant matrix. Here, one need to choose a reference point $z_{2}, r_{2}$ corresponding to the other point of Wilson line, and if $r_{2} \rightarrow \infty$, one can interpret the $z_{2}$ as the position of source on the boundary. We took limit $r_{2} \rightarrow \infty$ with factor $e^{2 h r_{2}}$ where the conformal dimension $h=\bar{h}$ of scalar field is given by

$$
h=\bar{h}=-\frac{N-1}{2} .
$$

The negative conformal dimension reflects that the semi-classical limit of higher spin $\mathrm{AdS}_{3} / \mathrm{CFT}_{2}$ is non-unitary [86, 105]. Furthermore, according to the dictionary of the higher spin $\mathrm{AdS}_{3} / \mathrm{CFT}_{2}[81,108]$, the scalar field in the $h s[\lambda]$ higher spin gravity has conformal dimension $h=\frac{1+\lambda}{2}$. In the semi-classical limit where we perform the analytic continuation $\lambda \rightarrow-N$, the conformal dimension of the scalar field becomes

$$
h=\frac{1+\lambda}{2} \quad \Longrightarrow \quad h=-\frac{N-1}{2} .
$$

This agrees with the conformal dimension of the master field.

In this prescription with Wilson line for the master field, it is natural to have "the highest weight state" due to the regularization. i.e.,

$$
c_{0} \equiv \lim _{r_{2} \rightarrow \infty} e^{2 h r_{2}} b\left(r_{2}\right) \tilde{c}_{0} b\left(r_{2}\right) \quad \Longrightarrow \quad\left(c_{0}\right)_{i j} \sim \delta_{i 1} \delta_{j 1}
$$

Furthermore, $\operatorname{tr}(C)$, represents the bulk-to-boundary propagator, and we also take limit $r_{1} \rightarrow \infty$ in $\operatorname{tr}(C)$, which leads to boundary-to-boundary propagator in the non-constant background. We take it as our bi-local field:

$$
\Phi_{\text {master }}^{\text {dressed }}\left(z_{1}, \bar{z}_{1} ; z_{2}, \bar{z}_{2}\right) \equiv \lim _{r \rightarrow \infty} e^{2 h r_{1}} \operatorname{tr}\left[C\left(r_{1}, z_{1}, \bar{z}_{1} ; z_{2}, \bar{z}_{2}\right)\right] .
$$

Recall that one can consider the soft mode expansion of the non-constant connection around a constant one. And, the boundary-to-boundary propagator in the non-constant background can be understood as the gravitationally dressed master field. Using the residual gauge transformation, we can express the dressed master field as follow.

$$
\begin{aligned}
& C^{\text {dressed }}\left(r_{1}, z_{1}, \bar{z}_{1} ; z_{2}, \bar{z}_{2}\right) \\
& \quad=b^{-1}\left(r_{1}\right) h^{-1}\left(z_{1}\right) e^{-a_{\text {BTZ }}\left(z_{1}-z_{2}\right)} h\left(z_{2}\right) c_{0} \bar{h}\left(\bar{z}_{2}\right) e^{-\bar{a}_{\text {BTZ }}\left(\bar{z}_{2}-\bar{z}_{1}\right)} \bar{h}^{-1}\left(\bar{z}_{1}\right) b^{-1}\left(r_{1}\right) .
\end{aligned}
$$


For the soft mode expansion

$$
\Phi_{\text {master }}^{\text {dressed }}\left(z_{1}, \bar{z}_{1} ; z_{2}, \bar{z}_{2}\right)=G\left(z_{1}, \bar{z}_{1} ; z_{2}, \bar{z}_{2}\right)+G^{(1)}\left(z_{1}, \bar{z}_{1} ; z_{2}, \bar{z}_{2}\right)+\cdots
$$

we use the infinitesimal residual gauge transformation in (2.34) parametrized by $\eta(z)$ and $\zeta(z)$ (See $(2.39)$ and $(2.40)$ ). The leading bi-local field $G\left(z_{1}, \bar{z}_{1} ; z_{2}, \bar{z}_{2}\right)$ is a boundary-toboundary propagator in the constant background:

$$
G\left(z_{1}, \bar{z}_{1} ; z_{2}, \bar{z}_{2}\right)=\left[\frac{\frac{\pi^{2}}{\tau \bar{\tau}}}{\sin \left(\frac{\pi z_{12}}{\tau}\right) \sin \left(\frac{\pi \bar{z}_{12}}{\bar{\tau}}\right)}\right]^{2 h} .
$$

For the sub-leading one, using the mode expansion of $\eta(z)$ and $\zeta(z)$ in (2.43), we have

$$
\frac{G^{(1)}\left(z_{1}, \bar{z}_{1} ; z_{2}, \bar{z}_{2}\right)}{G\left(z_{1}, \bar{z}_{1} ; z_{2}, \bar{z}_{2}\right)}=\sum_{|n| \geqq 2} \eta_{n} f_{2, n}\left(z_{1}, z_{2}\right)+\sum_{|n| \geqq 3} \zeta_{n} f_{3, n}\left(z_{1}, z_{2}\right)+(\text { anti-holomorphic }),
$$

where soft mode eigenfunction $f_{s, n}\left(z_{1}, z_{2}\right)(s=2,3)$ is found to be

$$
\begin{aligned}
& f_{2, n} \equiv \gamma_{2} e^{-\frac{2 \pi i n \chi}{\tau}}\left[n \cos \frac{2 \pi n \sigma}{\tau}-\frac{\sin \frac{2 \pi n \sigma}{\tau}}{\tan \frac{2 \pi \sigma}{\tau}}\right] \\
& f_{3, n} \equiv \gamma_{3} e^{-\frac{2 \pi i n \chi}{\tau}}\left[2 n^{2} \sin \frac{2 \pi n \sigma}{\tau}+6 n \frac{\cos \frac{2 \pi n \sigma}{\tau}}{\tan \frac{2 \pi \sigma}{\tau}}-2 \frac{1+2 \cos ^{2} \frac{2 \pi \sigma}{\tau}}{\sin ^{2} \frac{2 \pi \sigma}{\tau}} \sin \frac{2 \pi n \sigma}{\tau}\right]
\end{aligned}
$$

where we defined $(\chi, \sigma)$ in terms of the bi-local coordinates $\left(z_{1}, z_{2}\right)$ by

$$
\chi=\frac{1}{2}\left(z_{1}+z_{2}\right), \quad \sigma=\frac{1}{2}\left(z_{1}-z_{2}\right),
$$

and the coefficient $\gamma_{s}(s=2,3)$ is found to be

$$
\gamma_{2} \equiv-\frac{4 \pi i h}{\tau}, \quad \gamma_{3} \equiv \frac{4 \pi^{2} i h(2 h+1)}{\tau^{2}} .
$$

Also, we used the relation (2.33) derived from the smoothness of holonomy:

$$
\mathcal{L}_{0}=-\frac{k_{c s} \pi}{2 \tau^{2}}, \quad \overline{\mathcal{L}}_{0}=-\frac{k_{c s} \pi}{2 \bar{\tau}^{2}} .
$$

For propagators in the BTZ background, we need to impose the periodicity of the angular coordinate $\phi \sim \phi+2 \pi$, which can be realized by the method of images. This is also involved with the non-contractible cycle of BTZ black hole. We will discuss this issue in the next section together with Wilson line.

Remarks on the highest weight prescription. Before ending this section, we make a remark on the choice of "the highest weight state" $\left(c_{0}\right)_{i j} \sim \delta_{i 1} \delta_{j 1}$ from the point of view of the residual gauge transformation. From the solution in (3.10) of the master field in the constant background, the residual gauge transformation of the connection $a$ and $\bar{a}$ induces the transformation of $c_{0}$ where the information about the boundary operator $\left(z_{2}, \bar{z}_{2}\right)$ is 
encoded. To see this, note that the residual gauge transformation by $h$ will transform the $c_{0}$ as follow.

$$
c_{0} \quad \longrightarrow \quad h\left(z_{2}\right) c_{0} \bar{h}\left(\bar{z}_{2}\right) .
$$

For simplicity, let us consider the action of holomorphic gauge transformation, and we work out explicitly for $\operatorname{sl}(2)$ case with $\mathcal{L}_{0}=0$ :

$$
\begin{aligned}
\left(\mathbb{I}+\epsilon \lambda^{(1)}(z)\right)\left(\begin{array}{cc}
\left(c_{0}\right)_{11} & \left(c_{0}\right)_{12} \\
\left(c_{0}\right)_{21} & \left(c_{0}\right)_{22}
\end{array}\right)= & c_{0}+\left(c_{0}\right)_{11}\left(\begin{array}{cc}
-\frac{1}{2} f^{\prime}(z) & 0 \\
f(z) & 0
\end{array}\right)+\left(c_{0}\right)_{12}\left(\begin{array}{cc}
0 & -\frac{1}{2} f^{\prime}(z) \\
0 & f(z)
\end{array}\right) \\
& +\left(c_{0}\right)_{21}\left(\begin{array}{cc}
-\frac{1}{2} f^{\prime \prime}(z) & 0 \\
\frac{1}{2} f^{\prime}(z) & 0
\end{array}\right)+\left(c_{0}\right)_{22}\left(\begin{array}{cc}
0-\frac{1}{2} f^{\prime \prime}(z) \\
0 & \frac{1}{2} f^{\prime}(z)
\end{array}\right),
\end{aligned}
$$

where we parametrized the infinitesimal residual gauge parameter by

$$
\lambda^{(1)}(z)=\left(\begin{array}{cc}
-\frac{1}{2} f^{\prime}(z) & -\frac{1}{2} f^{\prime \prime}(z) \\
f(z) & \frac{1}{2} f^{\prime}(z)
\end{array}\right) .
$$

Recall the conformal transformation of primary $\phi$ and its descendant $L_{-1} \phi=\partial_{z} \phi$ by infinitesimal variation $\epsilon(z)$ is given by:

$$
\begin{aligned}
\delta_{\epsilon} \phi & =-\epsilon(z) \partial_{z} \phi-h \partial_{z} \epsilon(z) \phi \\
\delta_{\epsilon} \partial_{z} \phi & =-h \partial_{z}^{2} \epsilon(z) \phi-(h+1) \partial_{z} \epsilon(z) \partial_{z} \phi-\epsilon(z) \partial_{z}^{2} \phi,
\end{aligned}
$$

where $h$ is the conformal dimension of $\phi$. Comparing (3.28) with (3.31), the holomorphic part of $\left(c_{0}\right)_{11},\left(c_{0}\right)_{12}$ modes transform like primaries with $h=-\frac{1}{2}$ while $\left(c_{0}\right)_{21}$ and $\left(c_{0}\right)_{22}$ components behave like descendants. Also, note that the last term in (3.31) does not appear in the (3.28). This would be because the operator dual to the matter field in the semi-classical limit has the conformal dimension $h=-\frac{1}{2}$ and $\partial^{2} \phi$ is a null state for $\operatorname{SL}(2)$ case (See section 3.3). In the same way with anti-holomorphic transformation, one can show that $\left(c_{0}\right)_{11}$ and $\left(c_{0}\right)_{21}$ modes are primaries while $\left(c_{0}\right)_{12}$ and $\left(c_{0}\right)_{22}$ are descendants. Hence, $\left(c_{0}\right)_{11}$ mode is the primary operator in both holomorphic and anti-holomorphic sector, and this is why $\left(c_{0}\right)_{11}$ mode gives the correct bulk-to-boundary correlator. In section 3.3 , we will discuss how a special class of operators (e.g., the primary operator dual to scalar field in higher spin gravity) behave under $\mathcal{W}$ transformation. Based on this observation, one can see that "the highest state" mode indeed transforms as a primary for the case of $N=3$.

\subsection{Wilson line}

The Wilson line captures interesting physics in gauge theories. In the Chern-Simons gravity as a gauge theory, the Wilson line plays an important role. In [109-113], the Wilson line has been investigated in the Chern-Simons (higher spin) gravity to define entanglement entropy holographically. This proposal is very natural because Wilson line between two boundary points gives geodesic distance of them, which is equivalent to the Ryu-Takayanagi prescription $[114,115]$. In 2D, the Wilson line was studied in the Schwarzian theory to evaluate the OTOCs $[54,116,117]$. In previous section, the Wilson line also appears as a solution of the equation of motion of the master field. 
For Wilson line in Chern-Simons gravity, for fixed end points, one can still consider various Wilson line objects depending on the choice of states of the Wilson line operator. ${ }^{8}$ Those were extensively studied from the point of view of Ishibashi states in the dual $\mathrm{CFT}_{2}$ [113]. In this paper, we will simply consider the following Wilson line [110] between $\left(r_{1}, z_{1}, \bar{z}_{1}\right)$ and $\left(r_{2}, z_{2}, \bar{z}_{2}\right)$ as our bi-local field:

$$
\Phi_{\mathrm{Wilson}}\left(z_{1}, \bar{z}_{1} ; z_{2}, \bar{z}_{2}\right)=\lim _{r \rightarrow \infty} e^{4 h r} \operatorname{tr}\left[\mathcal{P} \exp \left(-\int_{r, z_{2}}^{r, z_{1}} A\right) \mathcal{P} \exp \left(-\int_{r, \bar{z}_{1}}^{r, \bar{z}_{2}} \bar{A}\right)\right],
$$

where we regularize Wilson line from boundary to boundary symmetrically. Then, this is equivalent to the definition of the gravitationally dressed master field in (3.17) with $\left(c_{0}\right)_{i j}=\delta_{i j}$. Hence, under the soft mode expansion, we have the same result.

$$
\Phi_{\text {Wilson }}^{\text {dressed }}\left(z_{1}, \bar{z}_{1} ; z_{2}, \bar{z}_{2}\right)=G\left(z_{1}, \bar{z}_{1} ; z_{2}, \bar{z}_{2}\right)+G^{(1)}\left(z_{1}, \bar{z}_{1} ; z_{2}, \bar{z}_{2}\right)+\cdots,
$$

where the leading and the sub-leading bi-locals are given by

$$
\begin{aligned}
G\left(z_{1}, \bar{z}_{1} ; z_{2}, \bar{z}_{2}\right) & =\left[\frac{\frac{\pi^{2}}{\tau \bar{\tau}}}{\sin \left(\frac{\pi z_{12}}{\tau}\right) \sin \left(\frac{\pi \bar{z}_{12}}{\bar{\tau}}\right)}\right]^{2 h}, \\
\frac{G^{(1)}\left(z_{1}, \bar{z}_{1} ; z_{2}, \bar{z}_{2}\right)}{G\left(z_{1}, \bar{z}_{1} ; z_{2}, \bar{z}_{2}\right)} & =\sum_{n} \eta_{n} f_{2, n}\left(z_{1}, z_{2}\right)+\zeta_{n} f_{3, n}\left(z_{1}, z_{2}\right)+(\text { anti-holomorphic) },
\end{aligned}
$$

with the same $f_{2, n}$ and $f_{3, n}$ as in the master field as before in (3.22) and (3.23). Though the mathematical definition of the simple Wilson line in our consideration is the same as that of the master field solution, the Wilson line can give geometric insights. First of all, the Wilson line will give the geodesic distance between two boundary points. In 3D, the geodesic corresponds to the Ryu-Takanayagi surface, and the Wilson line can provide the entanglement entropy [109, 110]. Following [110], we define the holographic entanglement entropy (on the constant Euclidean time slice) by

$$
S_{E E}(\Delta \phi) \equiv-\left.\frac{k_{c s}}{2 h} \log e^{4 h r_{0}} \Phi_{\text {Wilson }}^{\text {dressed }}\left(z_{1}, \bar{z}_{1} ; z_{2}, \bar{z}_{2}\right)\right|_{t_{E}=0, \phi=\phi_{1}-\phi_{2}},
$$

where $h$ is the conformal dimension of the operator at the end points of Wilson line. For our case, it is given by

$$
h=-\frac{1}{2}(N-1) .
$$

Also, we retrieved the UV divergence by the radial cutoff $r_{0}$ (See (3.32)). As pointed out in [110], this reproduces known entanglement entropy in the various 3D background. In particular, we are interested in BTZ background where the non-contractible cycle along $\phi$. For simplicity, we will consider the non-rotating BTZ black hole, ${ }^{9}$ and the Wilson line via (3.36) indeed reproduces the entanglement entropy in the BTZ background [110]

$$
S_{E E}(\Delta \phi)=\frac{c}{6} \log \left[\frac{\pi^{2} \beta^{2}}{l^{2} e^{-2 r_{0}}} \sinh ^{2}\left(\frac{\pi l \Delta \phi}{\beta}\right)\right] .
$$

\footnotetext{
${ }^{8}$ For instance, as in previous section one can take the trace of Wilson line operator. Or, we can also take a particular component. In fact, the solution of master field can be viewed as the highest weight state for $\left(z_{2}, \bar{z}_{2}\right)$ when we remove the trace by exchanging the holomorphic and anti-holomorphic part.

${ }^{9}$ See [7] for rotating BTZ black hole in SL(2) Chern-Simons gravity.
} 
Here, we used the relation between the central charge $c$ and Chern-Simons level $k_{c s}$

$$
c=6 k_{c s},
$$

and we also used the modular parameter of the non-rotating black hole:

$$
\tau=\frac{2 \pi i l}{r_{+}}=\frac{i \beta}{l}, \quad \bar{\tau}=-\frac{2 \pi i l}{r_{+}}=-\frac{i \beta}{l} .
$$

The existence of the non-contractible cycle could make Wilson line ambiguous. But, by considering the covering space of $\phi$, one can incorporate the contribution of horizon into Wilson line. First, let us consider $\phi=2 \pi$ where one end of the Wilson line rotate around whole boundary and comes back to the other point. in high temperature $\beta \gg l$, one can also recover the Bekenstein-Hawking formula for the BTZ black hole

$$
S_{E E}(2 \pi)=\frac{\pi r_{+}}{4 G}+\cdots,
$$

where we used $k_{c s}=\frac{l}{4 G}$. As the Wilson line winds the horizon further, it is natural to encode the winding number into bi-local fields like Wilson line as well as master field.

$$
\Phi_{m}^{\text {dressed }}\left(z_{1}, \bar{z}_{1} ; z_{2}, \bar{z}_{2}\right) \equiv \Phi^{\text {dressed }}\left(z_{1}+2 \pi m, \bar{z}_{1}+2 \pi m ; z_{2}, \bar{z}_{2}\right) \quad(m \in \mathbb{Z}) .
$$

For AdS vacuum where the modular parameter is given by $2 \pi$, the holonomy around the $\phi$ cycle is trivial. i.e., $\Phi_{m}^{\text {dressed }}\left(z_{1}, \bar{z}_{1} ; z_{2}, \bar{z}_{2}\right)=\Phi^{\text {dressed }}\left(z_{1}, \bar{z}_{1} ; z_{2}, \bar{z}_{2}\right)$, but it is not true for BTZ black hole. For non-constant background, one can also take the soft mode expansion of $\Phi_{m}^{\text {dressed: }}$

$$
\Phi_{m}^{\text {dressed }}\left(z_{1}, \bar{z}_{1} ; z_{2}, \bar{z}_{2}\right)=G_{m}\left(z_{1}, \bar{z}_{1} ; z_{2}, \bar{z}_{2}\right)+G_{m}^{(1)}\left(z_{1}, \bar{z}_{1} ; z_{2}, \bar{z}_{2}\right)+\cdots .
$$

When we evaluate the boundary-to-boundary propagator $G_{\mathrm{BTZ}}$ in the BTZ black hole background, we impose the periodicity in $\phi$ by hand by summing up those wound bilocals $[118,119]$

$$
G_{\mathrm{BTZ}}\left(z_{1}, \bar{z}_{1} ; z_{2}, \bar{z}_{2}\right)=\sum_{m} G_{m}\left(z_{1}, \bar{z}_{1} ; z_{2}, \bar{z}_{2}\right)=\sum_{m \in \mathbb{Z}} \frac{1}{\left[\sin \frac{\pi\left(z_{12}+2 \pi m\right)}{\tau} \sin \frac{\pi\left(\bar{z}_{12}+2 \pi m\right)}{\bar{\tau}}\right]^{2 h}} .
$$

Now, we will study the sub-leading term of $\Phi_{m}^{\text {dressed }}$ in the soft mode expansion:

$$
\begin{aligned}
G_{m}^{(1)}\left(z_{1}, \bar{z}_{1} ; z_{2}, \bar{z}_{2}\right)= & {\left[\sum_{|n| \geqq 2} \eta_{n} \tilde{f}_{2, n ; m}\left(z_{1}, z_{2}\right)+\sum_{|n| \geqq 3} \zeta_{n} \tilde{f}_{3, n ; m}\left(z_{1}, z_{2}\right)\right] G_{m}\left(z_{1}, \bar{z}_{1} ; z_{2}, \bar{z}_{2}\right) } \\
& +(\text { anti-holomorphic }),
\end{aligned}
$$

where $\tilde{f}_{2, n ; m}\left(z_{1}, z_{2}\right)$ and $\tilde{f}_{3, n ; m}\left(z_{1}, z_{2}\right)$ is found to be

$$
\begin{aligned}
& \tilde{f}_{2, n ; m} \equiv \gamma_{2} e^{-\frac{2 \pi i n \chi}{\tau}}\left[n \cos \frac{2 \pi n \sigma}{\tau}-\frac{\sin \frac{2 \pi n \sigma}{\tau}}{\left.\tan \frac{2 \pi(\sigma+\pi m)}{\tau}\right]}\right. \\
& \tilde{f}_{3, n ; m} \equiv \gamma_{3} e^{-\frac{2 \pi i n \chi}{\tau}}\left[2 n^{2} \sin \frac{2 \pi n \sigma}{\tau}+6 n \frac{\cos \frac{2 \pi n \sigma}{\tau}}{\tan \frac{2 \pi(\sigma+\pi m)}{\tau}}-2 \frac{1+2 \cos ^{2} \frac{2 \pi(\sigma+\pi m)}{\tau}}{\sin ^{2} \frac{2 \pi(\sigma+\pi m)}{\tau}} \sin \frac{2 \pi n \sigma}{\tau}\right],
\end{aligned}
$$


where $\gamma_{2}$ and $\gamma_{3}$ is given in (3.25). Note that for $m \neq 0$ we have

$$
\tilde{f}_{2,0, m}(\chi, \sigma)=\tilde{f}_{3,0, m}(\chi, \sigma)=0,
$$

while

$$
\tilde{f}_{2, \pm, m}(\chi, \sigma) \neq 0, \quad \tilde{f}_{3, \pm 1, m}(\chi, \sigma) \neq 0, \quad \tilde{f}_{3, \pm 2, m}(\chi, \sigma) \neq 0
$$

This reflects the well-known fact that in BTZ black hole the global $\operatorname{sl}(N)$ isometry is broken to its Cartan $[\mathrm{U}(1)]^{\oplus N-1}$.

\subsection{Null relation and Ward identity of $\mathcal{W}$-algebra}

In the last two sub-section, we have investigated the bi-local fields in the non-constant background and the soft mode expansion thereof. As we discussed, the soft mode, which generates (infinitesimal) residual gauge transformation, will induce the $\mathcal{W}$-transformations on the boundary. Hence, the soft mode expansion of the gravitationally dressed bi-local field give the Ward identity of $\mathcal{W}$-algebra on the boundary. In CFT, we know how two point function of any primary operators transforms under the conformal symmetry, and we have seen that soft mode expansion indeed reproduce the conformal transformation of two point function [11-16, 20]. In the previous section, we obtained soft mode expansion for $\mathcal{W}_{3}$ symmetry, which is related to the $\mathcal{W}_{3}$ transformation of two point function in CFT with $\mathcal{W}_{3}$ symmetry. However, from the point of view of CFT, this is, in general, difficult to study due to the nonlinearity of $\mathcal{W}$-algebra, and a general formula is not known at least to author's knowledge.

Though the $\mathcal{W}_{3}$ transformation of a generic primary would be difficult, one might be able to find it for a special case where non-linearity is suppressed. In this section, we derive the $\mathcal{W}_{s}$-transformation $(s=3,4)$ of a special primary based on its null relation in the 'tHooft limit of the $W_{N}$ minimal model $[108,120-122]$. Also, in appendix B we make a conjecture to extend the null relations and we derive the soft mode eigenfunctions for general spin $s$.

In $\mathcal{W}_{N}$ minimal model, a primary operator can be labelled by two Young tableaux $\left(\Lambda_{+} ; \Lambda_{-}\right)[81,108]$. In particular, a primary operator $\phi_{1} \equiv(\square ; 0)$ is dual to the scalar field in the dual higher spin gravity. It was shown [108] that because of null relation, the action of generators $W_{-n}^{(s)}$ on a primary operator $\phi_{1}$ of conformal dimension $h \equiv \frac{1}{2}(1+\lambda)$ is proportional to $L_{-1}^{n} \phi_{1}$ in large $c$. For instance, the null relation of $\phi_{1}$ was found to be

$$
\begin{aligned}
W_{0}^{(3)} \phi_{1} & =w^{(3)} \phi_{1}, \quad w^{(3)}=-(1+\lambda)(2+\lambda), \\
W_{-1}^{(3)} \phi_{1} & =\frac{3 w^{(3)}}{2 h} L_{-1} \phi_{1}, \\
W_{-2}^{(3)} \phi_{1} & =\frac{3 w^{(3)}}{h(2 h+1)} L_{-1}^{2} \phi_{1} .
\end{aligned}
$$


And, for its conjugate $\bar{\phi}_{1} \equiv(\bar{\square} ; 0)$, we have

$$
\begin{aligned}
W_{0}^{(3)} \bar{\phi}_{1} & =-w^{(3)} \bar{\phi}_{1}, \\
W_{-1}^{(3)} \bar{\phi}_{1} & =-\frac{3 w^{(3)}}{2 h} L_{-1} \bar{\phi}_{1}, \\
W_{-2}^{(3)} \bar{\phi}_{1} & =-\frac{3 w^{(3)}}{h(2 h+1)} L_{-1}^{2} \bar{\phi}_{1} .
\end{aligned}
$$

Note $\phi_{1}$ and $\bar{\phi}_{1}$ have opposite $W_{3}$ charge. Note that this primary operator corresponds to the master field and Wilson line in the previous section in the semi-classical limit.

At zero temperature, from the mode expansion of $W^{(s)}(z)$

$$
W^{(s)}(z)=\sum_{n} z^{-n-s} W_{n}^{(s)}
$$

the OPE of $W^{(s)}(z)$ with the primary field $\phi_{1}(w)$ defined via $W_{n}^{(s)} \phi_{1}=0, \forall n>0$ gives

$$
\begin{aligned}
-\frac{1}{2 \pi i} \oint d z \zeta^{(s)}(z) W^{(s)}(z) \phi_{1}(w) & =-\frac{1}{2 \pi i} \sum_{n} \oint d z \frac{\zeta^{(s)}(z)}{(z-w)^{n+s}}\left(W_{n}^{(s)} \phi_{1}\right)(w), \\
& =-\sum_{m=0}^{s-1} \frac{1}{m !} \partial_{z}^{m} \zeta^{(s)}(z)\left(W_{m-s+1}^{(s)} \phi_{1}\right)(w) .
\end{aligned}
$$

Using (3.50) (3.55) the Ward identity for $\mathcal{W}_{3}$ symmetry can be written as

$$
\begin{aligned}
& \delta_{\epsilon}\left\langle\phi_{1}\left(z_{1}\right) \bar{\phi}_{1}\left(z_{2}\right)\right\rangle \\
& \quad=-w^{(3)}\left[\frac{1}{2} \partial_{1}^{2} \zeta_{1}+\frac{3}{2 h} \partial_{1} \zeta_{1} \partial_{1}+\frac{3}{h(2 h+1)} \zeta_{1} \partial_{1}^{2}-(1 \rightarrow 2)\right]\left\langle\phi_{1}\left(z_{1}\right) \bar{\phi}_{1}\left(z_{2}\right)\right\rangle .
\end{aligned}
$$

where $\zeta_{i} \equiv \zeta\left(z_{i}\right)$ and $\partial_{i} \equiv \frac{\partial}{\partial z_{i}}(i=1,2)$. Then, inserting the two point function at zero temperature

$$
\left\langle\phi_{1}\left(z_{1}\right) \bar{\phi}_{1}\left(z_{2}\right)\right\rangle=\frac{1}{\left(z_{1}-z_{2}\right)^{2 h}},
$$

and using parametrization $\zeta(z)=\sum_{n} \zeta_{n} z^{n+2}$, we have the infinitesimal transformation of two point function under $\mathcal{W}_{3}$ symmetry as:

$$
\begin{aligned}
& \frac{\delta_{\epsilon}\left\langle\phi_{1}\left(z_{1}\right) \bar{\phi}_{1}\left(z_{2}\right)\right\rangle}{\left\langle\phi_{1}\left(z_{1}\right) \bar{\phi}_{1}\left(z_{2}\right)\right\rangle} \\
& \quad=w^{(3)} \sum_{n} \zeta_{n}\left[-\frac{1}{2} n^{2}\left(z_{1}^{n}-z_{2}^{n}\right)+\frac{3}{2} n \frac{z_{1}+z_{2}}{z_{1}-z_{2}}\left(z_{1}^{n}+z_{2}^{n}\right)-\frac{z_{1}^{2}+4 z_{1} z_{2}+z_{2}^{2}}{\left(z_{1}-z_{2}\right)^{2}}\left(z_{1}^{n}-z_{2}^{n}\right)\right] .
\end{aligned}
$$

At finite temperature, one can transform the zero temperature result in (3.60) by ${ }^{10}$

$$
z \quad \longrightarrow \quad e^{-i z}
$$

\footnotetext{
${ }^{10}$ Here, wee use $\tau=2 \pi$ for simplicity, and, we will recover it if necessary.
} 
or, one can also use the finite temperature representation of $L_{-1}$ in (3.57)

$$
L_{-1}=e^{i z}\left(\partial_{z}+i h\right) .
$$

Then, we can obtain

$$
\begin{aligned}
& \frac{\delta_{\epsilon}\left\langle\phi_{1}\left(z_{1}\right) \bar{\phi}_{1}\left(z_{2}\right)\right\rangle}{\left\langle\phi_{1}\left(z_{1}\right) \bar{\phi}_{1}\left(z_{2}\right)\right\rangle} \\
& \quad=\frac{i w^{(3)}}{2} \sum_{n} \epsilon_{n} e^{-i n\left(\frac{z_{1}+z_{2}}{2}\right)}\left[2 n^{2} \sin \frac{n z_{12}}{2}+6 n \frac{\cos \frac{n z_{12}}{2}}{\tan \frac{z_{12}}{2}}-2 \frac{1+2 \cos ^{2} \frac{z_{12}}{2}}{\sin ^{2} \frac{z_{12}}{2}} \sin \frac{n z_{12}}{2}\right],
\end{aligned}
$$

where $\epsilon_{i} \equiv \epsilon\left(z_{i}\right)$ and $\partial_{i}=\partial_{z_{i}}(i=1,2)$. This agrees with the soft mode eigenfunction derived from the soft mode expansion of the dressed bi-locals. In the same way, one can further obtain the transformation of two point function under $W^{(4)}$ symmetry. The null relations of $\phi_{1}$ are given by [108]

$$
\begin{aligned}
W_{0}^{(4)} \phi_{1} & =w^{(4)} \phi_{1}, \\
W_{-1}^{(4)} \phi_{1} & =\frac{4 w^{(4)}}{2 h} L_{-1} \phi_{1}, \\
W_{-2}^{(4)} \phi_{1} & =\frac{10 w^{(4)}}{2 h(2 h+1)} L_{-1}^{2} \phi_{1}, \\
W_{-3}^{(4)} \phi_{1} & =\frac{20 w^{(4)}}{2 h(2 h+1)(2 h+2)} L_{-1}^{3} \phi_{1},
\end{aligned}
$$

where $w^{(4)}$ denotes the $W_{0}^{(4)}$ charge of the operator $\phi_{1}$. This gives

$$
\begin{aligned}
\delta_{\zeta}\left\langle\phi_{1}\left(z_{1}\right) \bar{\phi}_{1}\left(z_{2}\right)\right\rangle & \\
=-w^{(4)}[ & {\left[\frac{1}{6} \partial_{1}^{3} \zeta_{1}+\frac{2}{2 h} \partial_{1}^{2} \zeta_{1} \partial_{1}+\frac{10}{2 h(2 h+1)} \partial_{1} \zeta_{1} \partial_{1}^{2}+\frac{20}{2 h(2 h+1)(2 h+2)} \zeta_{1} \partial_{1}^{3}\right.} \\
& +(1 \rightarrow 2)]\left\langle\phi_{1}\left(z_{1}\right) \bar{\phi}_{1}\left(z_{2}\right)\right\rangle .
\end{aligned}
$$

And, the transformation of two point function at finite temperature is found to be

$$
\begin{aligned}
& \frac{\delta_{\zeta}\left\langle\phi_{1}\left(z_{1}\right) \bar{\phi}_{1}\left(z_{2}\right)\right\rangle}{\left\langle\phi_{1}\left(z_{1}\right) \bar{\phi}_{1}\left(z_{2}\right)\right\rangle}=-\frac{w^{(4)}}{6} \sum_{n} \zeta_{n} e^{-i n\left(\frac{z_{1}+z_{2}}{2}\right)} \\
& \times {\left[2 n^{3} \cos \frac{n z_{12}}{2}-12 n^{2} \frac{\sin \frac{n z_{12}}{2}}{\tan \frac{z_{12}}{2}}+n\left(7-15 \cot ^{2} \frac{z_{12}}{2}-15 \csc ^{2} \frac{z_{12}}{2}\right) \cos \frac{n z_{12}}{2}\right.} \\
&\left.\quad+\left(5 \cot ^{3} \frac{z_{12}}{2}+25 \cot \frac{z_{12}}{2} \csc ^{2} \frac{z_{12}}{2}-7 \cot \frac{z_{12}}{2}\right) \sin \frac{n z_{12}}{2}\right] .
\end{aligned}
$$

In principle, one can confirm this result from $\mathrm{SL}(4, \mathbb{C})$ Chern-Simons gravity although it would be much more tedious calculation. However, from the null relation for $s=3,4$, we could find a pattern of the null relations, and we make a conjecture on null relation for general $s$ in appendix B. Namely, we conjecture that

$$
W_{-n}^{(s)} \phi_{1} \sim L_{-1}^{s} \phi_{1} .
$$


From this conjecture, we could obtain the soft mode eigenfunction for arbitrary $s$. Surprisingly, this result agrees with another conjecture on the recursion relation of those eigenfunctions. Based on the soft mode eigenfunction for $s=1,2$, [13] conjectured a criteria on the recursion relation to construct the eigenfunction for spin $s=n$ from those of $s=1,2, \cdots, n-1$. In section 3.4 , we complete the conjecture to find a concrete recursion relation to satisfy the criteria in [13]. And, the resulting eigenfunctions perfectly agree with those from the null relation. Furthermore, these eigenfunction satisfies a Casimir differential equation as expected, and we could finally find that those eigenfunctions have been observed as a basis in the large $q$ limit together with $\beta \mathcal{J} \rightarrow \infty$ limit of SYK model [20].

\subsection{Recursion relation of soft mode eigenfunction}

In general $\mathrm{SL}(N, \mathbb{C})$ Chern-Simon higher spin theory, it is practically difficult to derive the soft mode eigenfunction for arbitrary spin- $s$ explicitly. But, if there is underlying mathematical structure of those eigenfunctions, one might be able to derive them without referring to Chern-Simons higher spin gravity.

We begin with two eigenfunctions for $s=1,2$ [13]:

$$
\begin{aligned}
& f_{1, n}\left(z_{1}, z_{2}\right)=\frac{e^{-i n\left(\frac{z_{1}+z_{2}}{2}\right)}}{\sin \frac{z_{12}}{2}} \sin \frac{n z_{12}}{2}, \\
& f_{2, n}\left(z_{1}, z_{2}\right)=\frac{e^{-i n\left(\frac{z_{1}+z_{2}}{2}\right)}}{\sin \frac{z_{12}}{2}}\left[n \cos \frac{n z_{12}}{2}-\frac{\sin \frac{n z_{12}}{2}}{\tan \frac{z_{12}}{2}}\right],
\end{aligned}
$$

where we again use holomorphic sector only and choose the (inverse) temperature to be $\tau=2 \pi$ for simplicity. Note that we include $\sin \frac{z_{12}}{2}$ in the denominator which comes from the measure of the $\mathrm{SL}(2)$ invariant inner product [20,123]. It is also convenient to define the center of coordinate $\chi$ and the relative coordinate $\sigma$ by

$$
\chi \equiv \frac{1}{2}\left(z_{1}+z_{2}\right), \quad \sigma \equiv \frac{1}{2}\left(z_{1}-z_{2}\right) .
$$

By an abuse of notation, we will also redefine $f_{i, n}(\chi+\sigma, \chi-\sigma) \rightarrow f_{i, n}(\chi, \sigma)$ only in this section. We then have a simpler expression

$$
\begin{aligned}
& f_{1, n}(\chi, \sigma)=e^{-i n \chi} \frac{\sin n \sigma}{\sin \sigma}, \\
& f_{2, n}(\chi, \sigma)=\frac{e^{-i n \chi}}{\sin \sigma}\left[n \cos n \sigma-\frac{\sin n \sigma}{\tan \sigma}\right] .
\end{aligned}
$$

In the rest of this section we will find the modes $f_{s, n}$ for all $s, n$ by demanding certain conditions. From the translation symmetry of center of coordinate, which is a part of bi-local SL(2) symmetry, we expect

$$
f_{s, n}(\chi, \sigma) \equiv e^{-i n \chi} g_{s, n}(\sigma),
$$

for some $g_{s, n}(\sigma)$. For our purpose, we need to introduce two ingredients. First one is the $\mathrm{SL}(2)$ invariant inner product on the space of functions $f_{s, n}[20,123]$

$$
\left\langle f_{s, n}, f_{s^{\prime}, n^{\prime}}\right\rangle=\frac{1}{2 \pi} \int_{0}^{2 \pi} d \chi \int_{0}^{\pi} d \sigma f_{s, n}^{*}(\chi, \sigma) f_{s^{\prime}, n^{\prime}}(\chi, \sigma) .
$$


Note that we already absorbed the measure into $f_{s, n}$. This inner product can be naturally defined in the SYK model. Due to the translational symmetry of the center of coordinates, the inner product is diagonal in $n$-space. On the $g_{s, n}(z)$ function defined via (3.76), this inner product (for the same $n$ ) translates to

$$
\left\langle g_{s, n}, g_{s^{\prime}, n}\right\rangle=\int_{0}^{\pi} d \sigma g_{s, n}^{*}(\sigma) g_{s^{\prime}, n}(\sigma) .
$$

Another ingredient we need is a recursive way to build the functions $g_{s, n}(\sigma)$. Based on the observation that $f_{2, n}(\chi, \sigma)=\partial_{z} f_{1, n}(\chi, \sigma)$, it was conjectured in [13] that a mode $f_{s, n}$ is a linear combination of differential operators ${ }^{11} \partial_{\sigma}$ acting on modes $f_{s^{\prime}, n}$ for all $s^{\prime}<s$. We can make a reasonable conjecture that the set of functions $g_{s, n}(\sigma)$ satisfy the following two conditions:

$$
\begin{aligned}
I: & g_{s, n}(\sigma) & =\partial_{\sigma} g_{s-1, n}(\sigma)+\sum_{s^{\prime}=0}^{s-2} P_{s, s^{\prime}, n}\left(\partial_{\sigma}\right) g_{s^{\prime}, n}(\sigma), \\
I I: & \left\langle g_{s, n}, g_{s^{\prime}, n}\right\rangle & \sim \delta_{s, s^{\prime}} .
\end{aligned}
$$

where $P_{s, s^{\prime}, n}\left(\partial_{\sigma}\right)$ is a polynomial in $\partial_{\sigma}$. For the given $g_{1, n}(\sigma), g_{2, n}(\sigma)$ it is easy to find explicitly such polynomials $P_{s, s^{\prime}, n}\left(\partial_{\sigma}\right)$ satisfying (3.80) for low spins. For instance, we found that $[13]$

$$
\begin{aligned}
& g_{3, n}(\sigma)=\partial_{z} g_{2, n}(\sigma)+\frac{1}{3}\left(n^{2}-1\right) g_{1, n}(\sigma), \\
& g_{4, n}(z)=\partial_{z} g_{3, n}(\sigma)+\frac{4}{15}\left(n^{2}-4\right) g_{s, n}(\sigma),
\end{aligned}
$$

and so on. Continuing for a few more orders, we could guess that the correct recursion relation is

$$
g_{s+2, n}=\partial_{\sigma} g_{s+1, n}+F_{s, n} g_{s, n}
$$

where $F_{s, n}$ is defined by

$$
F_{s, n} \equiv \frac{s^{2}\left(n^{2}-s^{2}\right)}{4 s^{2}-1} .
$$

In addition, the initial data for the recursion relation are given by

$$
\begin{aligned}
& g_{1, n}(\sigma)=\frac{\sin n \sigma}{\sin \sigma}, \\
& g_{2, n}(\sigma)=\frac{1}{\sin \sigma}\left[n \cos n \sigma-\frac{\sin n \sigma}{\tan \sigma}\right] .
\end{aligned}
$$

In appendix C, we show that the solution of the recursion relation in (3.82) is found to be

$$
g_{s, n}(\sigma)=\left\{\begin{array}{ll}
\frac{[(s-1) !]^{2}}{(2 s-3) ! !} \frac{e^{i \pi s}}{\sin \sigma}\left(e^{i n \sigma} P_{s-1}^{n,-n}(\sigma)-e^{-i n \sigma} P_{s-1}^{-n, n}(\sigma)\right) & \text { for }|n| \geqq s \\
0 & \text { for }|n|<s
\end{array},\right.
$$

\footnotetext{
${ }^{11}$ From the point of view of bi-local map in the higher spin AdS/CFT, the relative coordinates $\sigma$ is related to the spin $s$ as well as radial coordinates of AdS.
} 
where $P_{n}^{\alpha, \beta}$ is a Jacobi Polynomial. Furthermore, we also show that $f_{s, n}$ 's are a solution of a second order differential equation:

$$
\left[-\partial_{\chi}^{2}+\partial_{\sigma}^{2}-\frac{s(s-1)}{\sin ^{2} \sigma}\right]\left[f_{s, n}(\chi, \sigma) \sin \sigma\right]=0 .
$$

Note that this is exactly the same as the differential equation found by [20] for the eigenfunction of the four point kernel in the large $q$ limit with $v \rightarrow 1$ of SYK model. Note that the $v \rightarrow 1$ limit corresponds to $\beta \mathcal{J} \rightarrow \infty$ limit in SYK model. In addition, in the zero temperature limit where $\sin \sigma$ is replaced by $\sigma$, such a differential equation is found in [104] as Casimir differential equation of the bi-local CFTs.

Note the function $f_{s, n}$ diagonalizes the translation generator of center of coordinates. From (3.87), one can easily show that $f_{s, n}$ are orthogonal. i.e.,

$$
\left\langle f_{s, n}, f_{s^{\prime}, n^{\prime}}\right\rangle=N_{s, n} \delta_{s, s^{\prime}} \delta_{n, n^{\prime}},
$$

where we found (See appendix C)

$$
N_{s, n} \equiv n \pi(2 s-1)\left[\frac{(s-1) !}{(2 s-1) ! !}\right]^{2} \prod_{j=1}^{s-1}\left(n^{2}-j^{2}\right) .
$$

\section{$4 \quad$ OTOC and Lyapunov exponent}

The Lyapunov exponent diagnosing the quantum chaos is found to be $\frac{2 \pi}{\beta}$ for the black hole background in the Einstein gravity. The maximal Lyapunov exponent comes from the graviton exchanges in the high energy scattering. When the scattering can be mediated by higher spin field in addition to graviton, the overall Lyapunov exponent could be changed because one can expect that the Lyapunov exponent by the exchange of the spin $s$ field ${ }^{12}$ is found to be [10]

$$
\lambda_{L}^{(s)}=\frac{2 \pi(s-1)}{\beta} .
$$

This seemingly violates the chaos bound. It was shown [10] that in $h s[\lambda]$ higher spin gravity which contains infinite tower of higher spin fields, the exponential growth coming from all higher spin fields forms a geometric series, and the overall Lyapunov exponent becomes zero. Because the $h s[\lambda]$ higher spin theories are unitary for $\lambda \in[0,1]$, the Lyapunov exponent should not exceed $\frac{2 \pi}{\beta}$. This is also consistent because the system with infinitely many conserved charge would not be chaotic. On the other hand, the $3 \mathrm{D}$ higher spin gravity in the semi-classical limit, the infinite tower of higher spin fields is truncated, and we have only a finite number of higher spin fields. Hence, the geometric series will terminate at a finite spin $s$, and therefore, it will violate the chaos bound. In fact, this is not surprising because this theory is not unitary.

For evaluation of the out-of-time-ordered correlator, we will start from Euclidean four point functions with a particular configuration. As we discussed in section 3, we also

\footnotetext{
${ }^{12}$ It was observed in [124] that the "pole-skipping" of $W_{3}$ current leads to the Lyapunov exponent $\frac{4 \pi}{\beta}$.
} 
consider four point function which can be evaluated by two point function of bi-local field (e.g., master field or Wilson line). In the non-constant background, we consider the two point function of the gravitationally dressed bi-local fields, and the soft mode expansion allow us to compute the higher spin contribution to the Euclidean correlator.

$$
\begin{aligned}
F(1,2,3,4) & \equiv\left\langle\Phi^{\text {dressed }}(1,2) \Phi^{\text {dressed }}(3,4)\right\rangle=G(1,2) G(3,4)+\left\langle G^{(1)}(1,2) G^{(1)}(3,4)\right\rangle+\cdots \\
& =G(1,2) G(3,4)+\sum_{s, n}\left\langle\zeta_{n}^{(s)} \zeta_{-n}^{(s)}\right\rangle\left[\delta_{\zeta_{n}^{(s)}} G(1,2)\right]\left[\delta_{\zeta_{-n}^{(s)}} G(3,4)\right]+\cdots
\end{aligned}
$$

where $\zeta_{n}^{(s)}$ is the spin $s$ soft mode. From the on-shell action, one can read off the two point function of soft modes, which is proportional to $\frac{1}{k_{c s}} \sim \frac{1}{c}$. Therefore, compared to the leading disconnected two point function, the contribution of higher spin field is suppressed by $\frac{1}{c}$, and this determine the scrambling time to be of order $\log c$.

In BTZ black hole, we also have to take the periodicity of the angle $\phi$ into account. For this, we consider the soft mode expansion of each image of the dressed bi-local field $\Phi_{m}^{\text {dressed }}\left(z_{1}, \bar{z}_{1} ; z_{2}, \bar{z}_{2}\right)$ in (3.42) due to the periodicity of $\phi \sim \phi+2 \pi$, and we denote it by $\tilde{f}_{n, m}$. For $N=3$ case, we have

$$
\begin{aligned}
& \tilde{f}_{2, n ; m}(\chi, \sigma ; \bar{\chi}, \bar{\sigma}) \equiv \delta_{\eta_{n}} \Phi_{m}^{\mathrm{dressed}} \\
&=\gamma_{2} G_{m}\left(z_{1}, z_{2} ; \bar{z}_{1}, \bar{z}_{2}\right) e^{-\frac{2 \pi i n \chi}{\tau}}\left[n \cos \frac{2 \pi n \sigma}{\tau}-\frac{\sin \frac{2 \pi n \sigma}{\tau}}{\tan \frac{2 \pi(\sigma+\pi m)}{\tau}}\right], \\
& \tilde{f}_{3, n ; m}(\chi, \sigma ; \bar{\chi}, \bar{\sigma}) \equiv \delta_{\zeta_{n}} \Phi_{m}^{\mathrm{dressed}} \\
&=\gamma_{3} G_{m}\left(z_{1}, z_{2} ; \bar{z}_{1}, \bar{z}_{2}\right) e^{-\frac{2 \pi i n \chi}{\tau}}\left[2 n^{2} \sin \frac{2 \pi n \sigma}{\tau}+6 n \frac{\cos \frac{2 \pi n \sigma}{\tau}}{\tan \frac{2 \pi(\sigma+\pi m)}{\tau}}\right. \\
&\left.-2 \frac{1+2 \cos ^{2} \frac{2 \pi(\sigma+\pi m)}{\tau}}{\sin ^{2} \frac{2 \pi(\sigma+\pi m)}{\tau}} \sin \frac{2 \pi n \sigma}{\tau}\right],
\end{aligned}
$$

where $\gamma_{2}$ and $\gamma_{3}$ are given in (3.25). It is useful to choose a particular configuration $\left(z_{1}, \bar{z}_{1} ; z_{2}, \bar{z}_{2}\right)=\left(\chi-\frac{\tau}{4}, \bar{\chi}-\frac{\bar{\tau}}{4} ; \chi+\frac{\tau}{4}, \bar{\chi}+\frac{\bar{\tau}}{4}\right)$ or equivalently $(\chi, \sigma, \bar{\chi}, \bar{\sigma})=\left(\chi,-\frac{\tau}{4}, \bar{\chi},-\frac{\bar{\tau}}{4}\right)$ in order to simplify the OTOC calculation [20]. With this choice of configuration, the leading one-point function of the bi-local becomes

$$
G_{m}\left(\sigma=-\frac{\tau}{4}, \bar{\sigma}=-\frac{\bar{\tau}}{4}\right)=\frac{1}{\left[\cos \frac{2 \pi^{2} m}{\tau} \cos \frac{2 \pi^{2} m}{\bar{\tau}}\right]^{2 h}} .
$$

Note that $G_{m}$ is independent of $\chi$ and $\bar{\chi}$, and we will parametrize it by $\sigma$ and $\bar{\sigma}$. i.e., $G_{m}(\sigma, \bar{\sigma})$. The soft mode eigenfunction $\tilde{f}_{s, n, m}=(s=2,3)$ can also be simplified as follow.

$$
\begin{aligned}
& \frac{\tilde{f}_{2, n, m}\left(\chi,-\frac{\tau}{4} ; \bar{\chi},-\frac{\bar{\tau}}{4}\right)}{G_{m}(-\tau / 4,-\bar{\tau} / 4)}=\gamma_{2} e^{-\frac{2 \pi i n \chi}{\tau}}\left[n \cos \frac{n \pi}{2}-\sin \frac{n \pi}{2} \tan \frac{2 \pi^{2} m}{\tau}\right], \\
& \frac{\tilde{f}_{3, n, m}\left(\chi,-\frac{\tau}{4} ; \bar{\chi},-\frac{\bar{\tau}}{4}\right)}{G_{m}(-\tau / 4,-\bar{\tau} / 4)}=\gamma_{3} e^{-\frac{2 \pi i n \chi}{\tau}}\left[-2\left(n^{2}+2-\frac{3}{\cos ^{2} \frac{2 m \pi^{2}}{\tau}}\right) \sin \frac{n \pi}{2}\right. \\
& \left.-6 n \cos \frac{n \pi}{2} \tan \frac{2 \pi^{2} m}{\tau}\right] \text {. }
\end{aligned}
$$


Note that the last term in (4.6) and (4.7) is odd in $m$. Hence, by summing them over $m$, those linear terms will be cancelled. But, the $m$ dependence of the first term in (4.7) still survives unlike $s=2$ case. Hence, the soft mode expansion of the dressed bi-locals for $s=2$ mode at $\sigma=-\frac{\tau}{4}$ can be factorized into a simple form

$$
\sum_{m} \delta_{\eta_{n}} \Phi_{m}^{\mathrm{dressed}}\left(\chi-\frac{\tau}{4}, \bar{\chi}-\frac{\bar{\tau}}{4} ; \chi+\frac{\tau}{4}, \bar{\chi}+\frac{\bar{\tau}}{4}\right)=\gamma_{2} e^{-\frac{2 \pi i n \chi}{\tau}} n \cos \frac{n \pi}{2} G_{\mathrm{BTZ}}(-\tau / 4,-\bar{\tau} / 4)
$$

while for $s=3$ case we have

$$
\begin{aligned}
& \sum_{m} \delta_{\zeta_{n}} \Phi_{m}^{\text {dressed }}\left(\chi-\frac{\tau}{4}, \bar{\chi}-\frac{\bar{\tau}}{4} ; \chi+\frac{\tau}{4}, \bar{\chi}+\frac{\bar{\tau}}{4}\right) \\
& \quad=-2 \gamma_{3} e^{-\frac{2 \pi i n \chi}{\tau}} \sum_{m}\left(n^{2}+2-3 \sec ^{2} \frac{2 m \pi^{2}}{\tau}\right) \sin \frac{n \pi}{2} G_{m}(-\tau / 4,-\bar{\tau} / 4) .
\end{aligned}
$$

We will evaluate the following OTOC which is regularized by thermal density matrix $\rho=e^{-\beta H}[5,20]$ :

$$
F(t, \phi)=\mathfrak{t r}\left[\rho^{\frac{1}{4}} V(0) \rho^{\frac{1}{4}} W(t, \phi) \rho^{\frac{1}{4}} V(0) \rho^{\frac{1}{4}} W(t, \phi)\right] .
$$

For this, we first evaluate the Euclidean correlator in (4.2) at the following configuration [20]:

$$
\begin{aligned}
& \left(z_{1}, \bar{z}_{1}\right)=\left(\chi-\frac{\tau}{4}, \bar{\chi}-\frac{\bar{\tau}}{4}\right) \\
& \left(z_{2}, \bar{z}_{2}\right)=\left(\chi+\frac{\tau}{4}, \bar{\chi}+\frac{\bar{\tau}}{4}\right) \\
& \left(z_{3}, \bar{z}_{3}\right)=(0,0) \\
& \left(z_{4}, \bar{z}_{4}\right)=\left(\frac{\tau}{2}, \frac{\bar{\tau}}{2}\right) .
\end{aligned}
$$

We evaluate the contribution of each spin- $s$ soft mode to the Euclidean four point function in $(4.2)$ :

$$
\left.\mathcal{F}_{s}(1,2,3,4) \equiv \frac{F(1,2,3,4)}{G_{\mathrm{BTZ}}(1,2) G_{\mathrm{BTZ}}(3,4)}\right|_{\text {spin-s }}=\sum_{n}\left\langle\zeta_{n}^{(s)} \zeta_{-n}^{(s)}\right\rangle \frac{\delta_{\zeta_{n}^{(s)}} G(1,2) \delta_{\zeta_{-n}^{(s)}} G(3,4)}{G_{\mathrm{BTZ}}(1,2) G_{\mathrm{BTZ}}(3,4)} .
$$

Here, we will use the two point function of soft modes from the on-shell action (e.g., (2.45) and (2.46)).

For the graviton contribution $(s=2)$, we have

$$
\begin{aligned}
\mathcal{F}_{2}(1,2,3,4) & =-\sum_{|n|=2,4,6, \cdots} \gamma_{2}^{2} \kappa_{2} \frac{(-1)^{\frac{n}{2}}}{n^{2}-1} e^{-\frac{2 \pi i n \chi}{\tau}}+(\text { anti-holomorphic }), \\
& =-\gamma_{2}^{2} \kappa_{2} \frac{1}{2 \pi i} \oint_{\mathcal{C}_{2}} d \zeta \frac{\frac{\pi}{2}}{\sin \frac{\pi \zeta}{2}} \frac{e^{-\frac{2 \pi i \zeta \chi}{\tau}}}{\zeta^{2}-1}+(\text { anti-holomorphic }),
\end{aligned}
$$




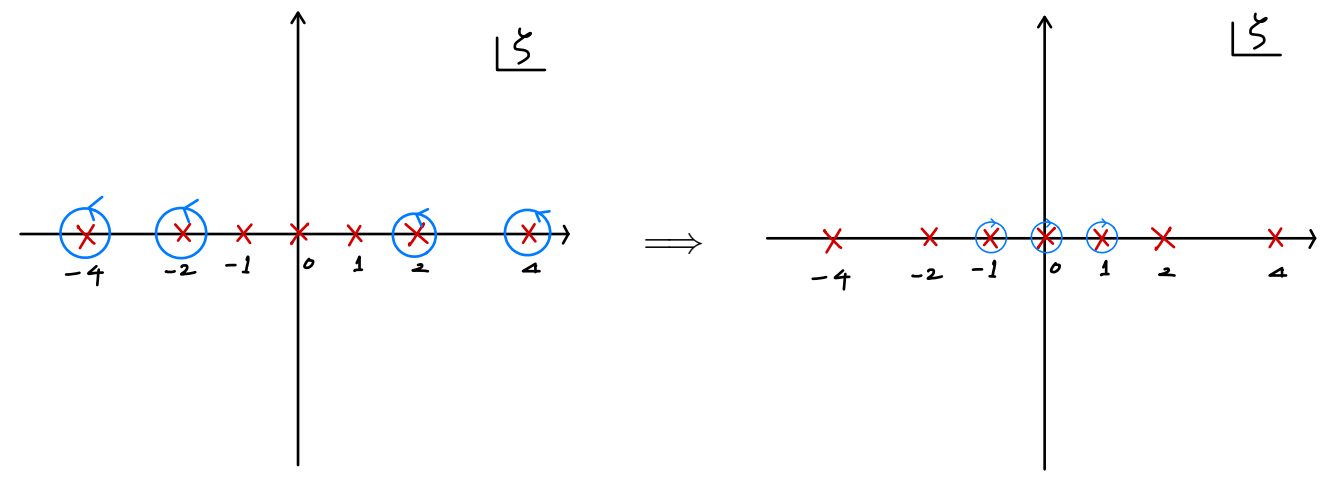

Figure 1. The contour integral for the graviton contribution.

where the summation runs over the even integer due to the special choice of the coordinates. In the second line, we rewrite the infinite series into a contour integral ${ }^{13}$ where the contour $\mathcal{C}_{2}$ is a collection of small counterclockwise circle around $\zeta= \pm 2, \pm 4, \cdots$. Note that the integrand also has a simple pole at $\zeta=0, \pm 1$. Hence, by pushing the contour to infinity, the contour integral becomes the summation of the residue at $\zeta=-1,0,1$ (See figure 1):

$$
\mathcal{F}_{2}(1,2,3,4)=\gamma_{2}^{2} \kappa_{2}\left[\frac{\pi}{4} e^{-\frac{2 \pi i \chi}{\tau}}-1+\frac{\pi}{4} e^{\frac{2 \pi i \chi}{\tau}}\right]+(\text { anti-holomorphic }) .
$$

With the Euclidean correlator, we will take the analytic continuation of the Euclidean time $t_{E}$ into the Lorentzian time $t$

$$
\chi=\phi+i \frac{t_{E}}{l}=\phi-\frac{t}{l},
$$

For simplicity, we will consider non-rotating BTZ black hole ${ }^{14}$ (embedded in higher spin gravity) where the modular parameter is given by ${ }^{15}$

$$
\tau=\frac{i \beta}{l}=\frac{2 \pi i l}{r_{+}}
$$

after the analytic continuation, we have

$$
\mathcal{F}_{2}(t, \phi)=-\frac{48 \beta}{\pi^{2} l c}\left[\frac{\pi}{4} e^{\frac{2 \pi}{\beta}(t-l \phi)}-1+\frac{\pi}{4} e^{-\frac{2 \pi}{\beta}(t-l \phi)}\right]+(\text { anti-holomorphic }),
$$

where we used $c=6 k_{c s}$.

\footnotetext{
${ }^{13}$ See [29] for clear explanation on the calculation in $[13,14,20]$ by using contour integral.

${ }^{14}$ See [7] for rotating BTZ black hole in SL(2) Chern-Simons gravity.

${ }^{15}$ The OTOCs of the rotating BTZ black hole in the Einstein gravity has been studied where one need "Boyer-Lindquist co-rotating frame" for the analytic continuation to real time [7].
} 
In contrast to the graviton contribution, the spin-3 field contribution has $m$ dependence even at the special coordinates that we focus on.

$$
\begin{aligned}
& \mathcal{F}_{3}(1,2,3,4) \\
& \equiv-\sum_{m, m^{\prime}} \sum_{|n|=3,5,7, \cdots} 4 i \gamma_{3}^{2} \kappa_{3} \\
& \quad \times \frac{(-1)^{\frac{n-1}{2}}\left(n^{2}+2-3 \sec ^{2} \frac{2 m \pi^{2}}{\tau}\right) \mathcal{A}_{m}\left(n^{2}+2-3 \sec ^{2} \frac{2 m^{\prime} \pi^{2}}{\tau}\right) \mathcal{A}_{m^{\prime}}}{n^{2}\left(n^{2}-1\right)\left(n^{2}-4\right)} e^{-\frac{2 \pi i n \chi}{\tau}} \\
& \quad+\text { (anti-holomorphic), }
\end{aligned}
$$

where we define

$$
\mathcal{A}_{m} \equiv \frac{G_{m}\left(-\frac{\tau}{4},-\frac{\bar{\tau}}{4}\right)}{G_{\mathrm{BTZ}}\left(-\frac{\tau}{4},-\frac{\bar{\tau}}{4}\right)} .
$$

For $m=0$, it is analogous to $s=2$ case where we can rewrite the infinite series as contour integral:

$$
\mathcal{F}_{3, m=0}(1,2,3,4)=-4 i \gamma_{3}^{2} \kappa_{3} \frac{1}{2 \pi i} \oint_{\mathcal{C}_{3}} d \zeta \frac{\frac{\pi}{2}}{\cos \frac{\pi \zeta}{2}} \frac{\zeta^{2}-1}{\zeta^{2}\left(\zeta^{2}-4\right)} e^{-\frac{2 \pi i \zeta \chi}{\tau}}+(\text { anti-holomorphic }),
$$

where the contour $\mathcal{C}_{3}$ is a collection of small counterclockwise circle at $\zeta= \pm 3, \pm 5, \cdots$. In the same way, we move the contour to pick up poles outside of the contour $\mathcal{C}_{3}$. Noting that the integrand has a simple pole at $\zeta= \pm 2$ and double pole at $\zeta=0$, we have

$$
\mathcal{F}_{3, m=0}(1,2,3,4)=4 i \gamma_{3}^{2} \kappa_{3}\left[-\frac{3 \pi}{32} e^{-\frac{4 \pi i \chi}{\tau}}-\frac{i \pi^{2}}{4 \tau} \chi+\frac{3 \pi}{32} e^{\frac{4 \pi i \chi}{\tau}}\right]+(\text { anti-holomorphic }) \text {. }
$$

For $m \neq 0$, one can write the infinite series into a similar contour integral:

$$
\begin{aligned}
& \mathcal{F}_{3, m \neq 0}(1,2,3,4) \\
& =-4 i \gamma_{3}^{2} \kappa_{3} \sum_{m, m^{\prime}} \frac{1}{2 \pi i} \oint_{\mathcal{C}_{3}} d \zeta \frac{\frac{\pi}{2}}{\cos \frac{\pi \zeta}{2}} \\
& \quad \times \frac{\left(\zeta^{2}-1-3 \tan ^{2} \frac{2 m \pi^{2}}{\tau}\right) \mathcal{A}_{m}\left(\zeta^{2}-1-3 \tan ^{2} \frac{2 m^{\prime} \pi^{2}}{\tau}\right) \mathcal{A}_{m^{\prime}}}{\zeta^{2}\left(\zeta^{2}-1\right)\left(\zeta^{2}-4\right)} e^{-\frac{2 \pi i \zeta \chi}{\tau}} \\
& \quad+\text { (anti-holomorphic). }
\end{aligned}
$$

Because of the $m$ dependence, the structure of poles could be changed for $m \neq 0$. We are interested, for simplicity, in (non-rotating) BTZ black hole where the modular parameter is pure imaginary. i.e., $\tan \left(\frac{2 m \pi^{2}}{\tau}\right)=i \tanh \left(\frac{2 m \pi^{2} l}{\beta}\right)$. Then, we have

$$
n^{2}-1 \leqq n^{2}-1+3 \tanh ^{2} \frac{2 m \pi^{2} l}{\beta}<n^{2}+2 .
$$

This implies that the $m$ dependence does not change the simple pole at $\zeta=2$ which will lead to the leading exponential growth after analytic continuation. ${ }^{16}$ Also, by moving the

\footnotetext{
${ }^{16}$ For some special $m$, the $\zeta=0$ pole could be changed.
} 
contour, the spin-3 contribution for all $m$ can be written as

$$
\begin{aligned}
\mathcal{F}_{3}(1,2,3,4)= & 4 i \gamma_{3}^{2} \kappa_{3}\left[-\frac{3 \pi}{8}\left(e^{-\frac{4 \pi i \chi}{\tau}}-e^{\frac{4 \pi i \chi}{\tau}}\right) \mathcal{A}^{2}+\cdots\right] \\
& +(\text { anti-holomorphic }),
\end{aligned}
$$

where $\mathcal{A}$ is defined by

$$
\mathcal{A} \equiv \sum_{m} p_{m} \mathcal{A}_{m} \quad \text { where } \quad p_{m} \equiv \frac{1}{2}\left(1-\tan ^{2} \frac{2 m \pi^{2}}{\tau}\right) .
$$

Here, we present only the contribution from the pole at $\zeta= \pm 2$. It is also easy to evaluate the contribution from other poles, but they will give the sub-leading exponential growth.

For (non-rotating) BTZ black hole, the modular parameter $\tau$ is pure imaginary ${ }^{17}$ so that we have

$$
0 \leqq p_{m} \leqq 1
$$

and as $m$ becomes larger, $p_{m}$ approaches to 1 . Note that $\mathcal{A}$ can be viewed as averaged $p_{m}$ weighted by $G_{m}$. Using (4.5), one can easily estimate that $\mathcal{A}$ approaches to 1 for $h<0$ which is the conformal dimension of our bi-locals. In the contributions of other poles, which include $e^{ \pm \frac{2 \pi i \chi}{\tau}} \chi e^{ \pm \frac{2 \pi i \chi}{\tau}} \chi$, also have a similar $m$ dependence. e.g.,

$$
\sum_{m} \tan ^{2} \frac{2 m \pi^{2}}{\tau} \mathcal{A}_{m}, \quad \sum_{m} \sec ^{2} \frac{2 m \pi^{2}}{\tau} \mathcal{A}_{m}, \quad \sum_{m} \sec ^{2} \frac{2 m \pi^{2}}{\tau} \tan ^{2} \frac{2 m \pi^{2}}{\tau} \mathcal{A}_{m} .
$$

And, they would also become of order 1 or vanish. After analytic continuation, the leading exponential growth in real time in the spin-3 contribution is

$$
\mathcal{F}_{3}(1,2,3,4)=\frac{432 i \beta}{\pi c l}\left[\mathcal{A} e^{\frac{4 \pi}{\beta}(t-l \phi)}+\cdots\right]+(\text { anti-holomorphic }) .
$$

And, the leading Lyapunov exponent is $\frac{4 \pi}{\beta}$.

\section{Conclusion}

In this paper, we investigated the quantum chaos in $\operatorname{SL}(N, \mathcal{C})$ Chern-Simons higher spin gravity. With the boundary term and the corresponding boundary condition in [96], we have derived the quadratic on-shell action of the smooth higher spin modes in $\operatorname{SL}(N, \mathbb{C})$ Chern-Simons higher spin gauge theories in $\mathrm{EAdS}_{3}$. We considered the gravitational dressing of bi-local objects such as the matter master field in $\operatorname{SL}(N, \mathcal{C})$ Vasiliev equation and Wilson line in the non-constant background. From the soft higher spin mode expansion of the dressed bi-locals, we have obtained the soft mode eigenfunction. In addition, based on the null relations in $\mathcal{W}_{N}$ minimal model, we have shown that the Ward identity of $\mathcal{W}$ symmetry leads to the same eigenfunction. We have also constructed a recursion relation of the soft mode eigenfunctions, and we found the form of the eigenfunction for all spin $s$.

\footnotetext{
${ }^{17}$ The AdS vacuum has real modular parameter. i.e., $\tau=2 \pi$. But, in this case, the angular coordinate is already periodic, and we don't have to sum up those images.
} 
Using the on-shell action and the soft mode eigenfunction, we have evaluated the OTOCs and have explicitly shown that the Lyapunov exponent of the SL(3) Chern-Simons higher spin gravity is $\frac{4 \pi}{\beta}$. In appendix D, we also evaluate OTOCs for the $\operatorname{SL}(N)$ case and the Lyapunov exponent is found to be $\frac{2 \pi}{\beta}(N-1)$.

For $N>2$, the $\operatorname{SL}(N)$ Chern-Simons higher spin gravity violates the chaos bound, which reflect that it is not unitary. The $\mathrm{SL}(N)$ higher spin gravity can be considered as the semi-classical limit of $h s[\lambda]$ higher spin gravity which is unitary for $\lambda \in[0,1]$. The $h s[\lambda]$ higher spin gravity has infinite tower of higher spin fields, and it was shown in [10] that its Lyapunov exponent is zero. In the semi-classical limit $(\lambda \rightarrow-N)$, the infinite tower of higher spin fields is truncated to a finite number of higher spin fields, and at the same time, it becomes non-unitary. We have observed the non-unitarity in the negative conformal dimension of the scalar field and the Wilson line discussed in section 3.

It is also interesting to consider a possibility of the higher spin generalization of the chaos bound. The 2D and 3D gravity can have a finite number of interacting higher spin fields at the cost of losing unitarity and causality [10]. Therefore, in the proof of the bound on chaos, the argument based on the unitarity and the causality will not hold, which leads to the violation of the chaos bound. In spite of the violation of the bound, the presence of a finite number of higher spin fields seemingly suggest a larger bound on Lyapunov exponent. It would be interesting to prove a new bound on chaos in the non-unitary field theories ${ }^{18}$ of which bulk dual has a finite number of higher spin fields.

In this work, we have considered the simplest constant background without spin-3 chemical potential. Therefore, the on-shell action has only the spin- 2 charge $\mathcal{L}$. If we turn on the spin- 3 chemical potential, it will generate the spin-3 charge $\mathcal{W}$ in the on-shell action. Then, the quadratic action contains the interaction between the spin- 2 and spin- 3 soft modes:

$$
\int \frac{d z^{2}}{2 \pi \operatorname{Im}(\tau)} \mathcal{W} \sim \sum_{n \in \mathbb{Z}} n^{2}\left(n^{2}-1\right)\left(n^{2}-4\right) f_{-n} g_{n}
$$

This is analogous to the coupling between $\mathrm{U}(1)$ and reparametrization modes in complex SYK model and 2D gravity [125]. It was reported in [90] that the presence of the spin-3 chemical potential can change entanglement entropy negative, which leads to the bound on spin-3 chemical potential. It will be interesting to investigate the quantum chaos with the coupling between spin-2 and spin-3 soft mode.

In [124], it was pointed out ${ }^{19}$ that the "pole-skipping" phenomenon [124, 126-129] of the $W_{3}$ higher spin current can capture the Lyapunov exponent $\frac{4 \pi}{\beta}$. These techniques might provide alternate ways to understanding the Lyapnov exponent in the context of higher spin holography.

\section{Acknowledgments}

We would like to thank Antal Jevicki, Matthias R. Gaberdiel, Mikhail A. Vasiliev, Spenta Wadia, Robert de Mello Koch, Euihun Joung, Thomas Basile, Keun-Young Kim, Viktor

\footnotetext{
${ }^{18}$ Recently, it was reported that the non-unitary Fishnet model violates the bound on chaos [58].

${ }^{19}$ We thank Felix Haehl for bringing this to our attention.
} 
Jahnke, Sangjin Sin, Dario Rosa and especially Ioannis Papadimitriou for extensive discussions. JY thanks the Korea Institute for Advanced Study (KIAS) for the hospitality and support during the initial stages of this work, within the program "IPMU-KIAS-Kyunghee Univ. joint workshop 2017". JY thank the Yukawa Institute for Theoretical Physics at Kyoto University for generous support during the course of this work, within the workshop YITP-T-18-04 "New Frontiers in String Theory 2018". JY thank the Kavli Institute for Theoretical Physics for generous support during the course of this work, within the workshop "Chaos and Order: from strongly correlated systems to black holes 2018". This research was supported in part by the National Science Foundation under Grant No. NSF PHY-1748958. JY thank the Okinawa Institute of Science and Technology (OIST) for the hospitality and generous support during the course of this work, within the workshop "OIST Mini Symposium, Holographic Tensors 2018". JY thanks the Erwin Schrodinger International Institute (ESI) where this work was completed during the program "Higher Spins and Holography 2019", and JY would like to thank the organizers for giving an opportunity to present our work.

\section{A Conventions}

In this paper, we use the following representation for the generators of $\operatorname{sl}(3)$ :

$$
\begin{aligned}
& L_{1}=\left(\begin{array}{ccc}
0 & 0 & 0 \\
\sqrt{2} & 0 & 0 \\
0 & \sqrt{2} & 0
\end{array}\right), \quad L_{0}=\left(\begin{array}{ccc}
1 & 0 & 0 \\
0 & 0 & 0 \\
0 & 0 & -1
\end{array}\right), \quad L_{-1}=\left(\begin{array}{ccc}
0 & -\sqrt{2} & 0 \\
0 & 0 & -\sqrt{2} \\
0 & 0 & 0
\end{array}\right) \text {, } \\
& W_{2}=\left(\begin{array}{ccc}
0 & 0 & 0 \\
0 & 0 & 0 \\
4 & 0 & 0
\end{array}\right), \quad W_{1}=\left(\begin{array}{ccc}
0 & 0 & 0 \\
\sqrt{2} & 0 & 0 \\
0 & -\sqrt{2} & 0
\end{array}\right), \quad W_{0}=\frac{2}{3}\left(\begin{array}{ccc}
1 & 0 & 0 \\
0 & -2 & 0 \\
0 & 0 & 1
\end{array}\right) \text {, } \\
& W_{-1}=\left(\begin{array}{ccc}
0 & -\sqrt{2} & 0 \\
0 & 0 & \sqrt{2} \\
0 & 0 & 0
\end{array}\right), \quad W_{-2}=\left(\begin{array}{ccc}
0 & 0 & 4 \\
0 & 0 & 0 \\
0 & 0 & 0
\end{array}\right)
\end{aligned}
$$

and, their traces are

$$
\operatorname{tr}\left(L_{n} L_{-m}\right)=\left(\begin{array}{ccc}
0 & 0 & -4 \\
0 & 2 & 0 \\
-4 & 0 & 0
\end{array}\right), \quad \operatorname{tr}\left(W_{n} W_{-m}\right)=\left(\begin{array}{ccccc}
0 & 0 & 0 & 0 & 16 \\
0 & 0 & 0 & -4 & 0 \\
0 & 0 & \frac{8}{3} & 0 & 0 \\
0 & -4 & 0 & 0 & 0 \\
16 & 0 & 0 & 0 & 0
\end{array}\right) .
$$

Note that this trace tr is the summation over the fundamental representation. The normalized trace $\operatorname{Tr}$ is defined by

$$
\operatorname{Tr}(A)=\frac{1}{\operatorname{tr}\left(L_{0} L_{0}\right)} \operatorname{tr}(A) .
$$

For $\mathrm{SL}(2, \mathbb{C})$ Chern-Simons gravity, the representations of the generators of $\operatorname{sl}(2)$ are

$$
L_{1}=\left(\begin{array}{ll}
0 & 0 \\
1 & 0
\end{array}\right), \quad L_{0}=\left(\begin{array}{cc}
\frac{1}{2} & 0 \\
0 & -\frac{1}{2}
\end{array}\right), \quad L_{-1}=\left(\begin{array}{cc}
0 & -1 \\
0 & 0
\end{array}\right) .
$$




\section{B Conjecture on null relation and $\mathcal{W}$ transformation}

In this appendix, we make a conjecture generalizing the null relation discussed in section 3.3. Based on this conjecture, we derive the infinitesimal transformation of two point function under the $W_{s}$ transformation.

In large $c$ limit of $\mathcal{W}_{N}$ minimal model, we conjecture that for a primary operator $\phi$, the action of $W_{-n}^{(s)}$ on $\phi$ will be proportional to $\left(L_{-1}\right)^{n} \phi$ in large $c$ limit:

$$
W_{-n}^{(s)} \phi \sim\left(L_{-1}\right)^{n} \phi+\mathcal{O}(1 / c)
$$

Note that in this conjecture we consider special primary operator in $\mathcal{W}_{N}$ minimal model such as $(\square ; 0),(\boxminus ; 0)$ etc.. But, this conjecture might hold in a large class of primary operators in $\mathcal{W}_{N}$ minimal model (e.g., simple primaries constructed on the Fock space found in [120-122]). Motivated by the explicit expressions for the low $s$, we consider the following ansatz.

$$
W_{-n}^{(s)} \phi=\frac{a_{n} w^{(s)}}{\prod_{j=0}^{n-1}(2 h+j)}\left(L_{-1}\right)^{n} \phi
$$

where $h$ and $w^{(s)}$ denotes the $L_{0}$ and $W_{0}^{(s)}$ charge of the primary $\phi$, respectively. i.e., we will choose $a_{0}=1$. Here, we chose To find $a_{n}$, we act $L_{1}$ on the both sides of (B.2). Using the commutation relation

$$
\left[L_{m}, W_{n}^{(s)}\right]=((s-1) m-n) W_{m+n},
$$

we have

$$
L_{1} W_{-n}^{(s)} \phi=(s-1+n) W_{-n+1}^{(s)} \phi=\frac{a_{n} w^{(s)}}{\prod_{j=0}^{n-1}(2 h+j)} L_{1} L_{-1}^{n} \phi=\frac{n a_{n} w^{(s)}}{\prod_{j=0}^{n-2}(2 h+j)} L_{-1}^{n-1} \phi .
$$

This leads to recursion relation of $a_{n}$, and we have

$$
a_{n}=\frac{s-1+n}{n} a_{n-1}=\cdots=\frac{(s-1+n) !}{n !(s-1) !} a_{0}=\frac{(s-1+n) !}{n !(s-1) !}
$$

Hence, based on our conjecture, the action of $W_{-n}^{(s)}$ on the primary operator $\phi$ in large $c$ is found to be

$$
W_{-n}^{(s)} \phi=\frac{(s-1+n) !}{n !(s-1) !} \frac{(2 h+n) w^{(s)}}{\prod_{j=0}^{n}(2 h+j)}\left(L_{-1}\right)^{n} \phi .
$$

Note that for the given $\mathcal{W}_{s}$ charge of $\phi$, the $\mathcal{W}_{s}$ charge of the conjugate $\bar{\phi}$ is given by

$$
W_{0}^{(s)} \phi=w^{(s)} \phi \quad \Longrightarrow \quad W_{0}^{(s)} \bar{\phi}=(-1)^{s} w^{(s)} \bar{\phi}
$$


Using the action of $W_{-n}^{(s)}$ generators on the primary, one can find the $\mathcal{W}_{s}$ transformation of two point function at zero temperature as in section 3.3:

$$
\begin{aligned}
& \frac{\delta_{\epsilon}\left\langle\phi\left(z_{1}\right) \bar{\phi}\left(z_{2}\right)\right\rangle}{\left\langle\phi\left(z_{1}\right) \bar{\phi}\left(z_{2}\right)\right\rangle} \\
& =-\sum_{m=0}^{s-1} \frac{1}{(s-1-m) !}\left(\partial_{1}^{s-1-m} \epsilon\left(z_{1}\right)\left\langle\left(W_{-m}^{(s)} \phi\right)\left(z_{1}\right) \phi\left(z_{2}\right)\right\rangle+\partial_{2}^{s-1-m} \epsilon\left(z_{2}\right)\left\langle\phi\left(z_{1}\right)\left(W_{-m}^{(s)} \phi\right)\left(z_{2}\right)\right\rangle\right), \\
& =-\frac{w^{(s)}}{(s-1) !} \sum_{n} \epsilon_{n} \sum_{m=0}^{s-1} \frac{(n+s-1) !(s+m-1) !}{(n+m) ! m !(s-m-1) !}(-1)^{m} \frac{z_{1}^{m+n}+(-1)^{m+s} z_{2}^{m+n}}{\left(z_{1}-z_{2}\right)^{m}},
\end{aligned}
$$

where we expanded $\epsilon(z)$ as

$$
\epsilon(z)=\sum_{n} z^{n+s-1} \epsilon_{n}
$$

Using the transformation into finite temperature ${ }^{20}$

$$
z \quad \Longrightarrow \quad e^{-i z}
$$

we have

$$
\begin{gathered}
\frac{\delta_{\epsilon}\left\langle\phi\left(z_{1}\right) \bar{\phi}\left(z_{2}\right)\right\rangle}{\left\langle\phi\left(z_{1}\right) \bar{\phi}\left(z_{2}\right)\right\rangle}=-\frac{w^{(s)}}{(s-1) !} \sum_{n} \epsilon_{n} e^{-i n \frac{z_{1}+z_{2}}{2}} \sum_{m=0}^{s-1} \frac{(n+s-1) !(s+m-1) !}{(n+m) ! m !(s-m-1) !}(-1)^{s} \\
\times \frac{\left(e^{i(m+n) \frac{z_{1}-z_{2}}{2}}+(-1)^{m+s} e^{-i(m+n) \frac{z_{1}-z_{2}}{2}}\right)}{\left(-2 i \sin \frac{z_{1}-z_{2}}{2}\right)^{m}}, \\
=-\frac{w^{(s)}}{(s-1) !} \sum_{n} \epsilon_{n} e^{-i n \chi} \frac{(n+s-1) !}{n !} \\
\times\left[(-1)^{s} e^{i n \sigma}{ }_{2} F_{1}\left(1-s, s, 1+n, \frac{e^{i \sigma}}{e^{i \sigma}-e^{-i \sigma}}\right)\right. \\
\left.+e^{-i n \sigma}{ }_{2} F_{1}\left(1-s, s, 1+n,-\frac{e^{-i \sigma}}{e^{i \sigma}-e^{-i \sigma}}\right)\right] .
\end{gathered}
$$

For $s>|n|$, the above expression vanishes because

$$
\begin{aligned}
& (-1)^{s} e_{2}^{i n z} F_{1}\left(1-s, s, 1+n, \frac{e^{i \sigma}}{e^{i \sigma}-e^{-i \sigma}}\right)+e_{2}^{-i n \sigma} F_{1}\left(1-s, s, 1+n,-\frac{e^{-i \sigma}}{e^{i \sigma}-e^{-i \sigma}}\right) \\
& \quad \propto\left[(-1)^{s}(-1)^{\frac{n}{2}} P_{s-1}^{-n}(i \cot [\sigma])+(-1)^{-\frac{n}{2}} P_{s-1}^{-n}(-i \cot [\sigma])\right] \\
& \quad=0 .
\end{aligned}
$$

Of course, this is exactly agree with what we expect. Namely, the soft mode eigenfunction $f_{s, n}$ vanishes when it is involved with global subalgebra i.e., $|n|<s$. For $s \leqq|n|$, one can

\footnotetext{
${ }^{20}$ In the appendix, we also use $\tau=2 \pi$ unit for simplicity, and, we will recover it if necessary.
} 
rewrite (B.12) in terms of Jacobi polynomial:

$$
\begin{aligned}
& \frac{\delta_{\epsilon}\langle\phi(\chi+\sigma) \bar{\phi}(\chi-\sigma)\rangle}{\langle\phi(\chi+\sigma) \bar{\phi}(\chi-\sigma)\rangle} \\
& \quad=-\frac{w^{(s)}}{(s-1) !} \sum_{n} \epsilon_{n} e^{-i n \chi}(s-1) !(-1)^{s}\left[e^{i n \sigma} P_{s-1}^{n,-n}(i \cot \sigma)-e^{-i n \sigma} P_{s-1}^{-n, n}(i \cot \sigma)\right] .
\end{aligned}
$$

To see the relation to the eigenfunction in large $q$ limit (together with $v \rightarrow 1$ limit) found in [20], we rewrite them using the transformation of hypergeometric function:

$$
\begin{aligned}
f_{s, n}(\chi, \sigma)= & e^{-i n \chi} \frac{(n+s-1) !}{n !}\left[(-1)^{s} e^{i n \sigma}{ }_{2} F_{1}\left(1-s, s, 1+n, \frac{e^{i \sigma}}{e^{i \sigma}-e^{-i \sigma}}\right)\right. \\
& \left.+e^{-i n \sigma}{ }_{2} F_{1}\left(1-s, s, 1+n,-\frac{e^{-i \sigma}}{e^{i \sigma}-e^{-i \sigma}}\right)\right], \\
= & \sqrt{\pi} e^{-i n \chi}(n+s-1) !(-1)^{s} e^{\frac{\pi i n}{2}} \frac{(\sin \sigma)^{s}}{s^{n}} \\
\times & {\left[\frac{{ }_{2} F_{1}\left(\frac{s+n}{2}, \frac{s-n}{2}, \frac{1}{2}, \cos ^{2} \sigma\right)}{\Gamma\left(\frac{n+s+1}{2}\right) \Gamma\left(\frac{n-s+2}{2}\right)}-2 i \cos \sigma \frac{{ }_{2} F_{1}\left(\frac{s+n+1}{2}, \frac{s-n+1}{2}, \frac{3}{2}, \cos ^{2} \sigma\right)}{\Gamma\left(\frac{n+s}{2}\right) \Gamma\left(\frac{n-s+1}{2}\right)}\right] } \\
+ & \sqrt{\pi} e^{-i n \chi}(n+s-1) ! e^{-\frac{\pi i n}{2}} \frac{(\sin \sigma)^{s}}{s^{n}} \\
& \times\left[\frac{{ }_{2} F_{1}\left(\frac{s+n}{2}, \frac{s-n}{2}, \frac{1}{2}, \cos ^{2} \sigma\right)}{\Gamma\left(\frac{n+s+1}{2}\right) \Gamma\left(\frac{n-s+2}{2}\right)}+2 i \cos \sigma \frac{2 F_{1}\left(\frac{s+n+1}{2}, \frac{s-n+1}{2}, \frac{3}{2}, \cos ^{2} \sigma\right)}{\Gamma\left(\frac{n+s}{2}\right) \Gamma\left(\frac{n-s+1}{2}\right)}\right] .
\end{aligned}
$$

For even $s$, one can simplify them as

$$
\begin{aligned}
& f_{s, n}(\chi, \sigma)=2 \sqrt{\pi} e^{-i n \chi}(n+s-1) !(-1)^{s} \frac{(\sin \sigma)^{s}}{s^{n}} \\
& \times\left[\cos \frac{\pi i n}{2} \frac{{ }_{2} F_{1}\left(\frac{s+n}{2}, \frac{s-n}{2}, \frac{1}{2}, \cos ^{2} \sigma\right)}{\Gamma\left(\frac{n+s+1}{2}\right) \Gamma\left(\frac{n-s+2}{2}\right)}+2 \sin \frac{\pi i n}{2} \cos z \frac{{ }_{2} F_{1}\left(\frac{s+n+1}{2}, \frac{s-n+1}{2}, \frac{3}{2}, \cos ^{2} \sigma\right)}{\Gamma\left(\frac{n+s}{2}\right) \Gamma\left(\frac{n-s+1}{2}\right)}\right] .
\end{aligned}
$$

For odds, we have

$$
\begin{aligned}
& f_{s, n}(\chi, \sigma)=2 \sqrt{\pi} e^{-i n \chi}(n+s-1) !(-1)^{s} \frac{(\sin \sigma)^{s}}{s^{n}} \\
& \times\left[\sin \frac{\pi i n}{2} \frac{2 F_{1}\left(\frac{s+n}{2}, \frac{s-n}{2}, \frac{1}{2}, \cos ^{2} \sigma\right)}{\Gamma\left(\frac{n+s+1}{2}\right) \Gamma\left(\frac{n-s+2}{2}\right)}-2 \cos \frac{\pi i n}{2} \cos \sigma \frac{2 F_{1}\left(\frac{s+n+1}{2}, \frac{s-n+1}{2}, \frac{3}{2}, \cos ^{2} \sigma\right)}{\Gamma\left(\frac{n+s}{2}\right) \Gamma\left(\frac{n-s+1}{2}\right)}\right] .
\end{aligned}
$$

\section{Recursion relation, orthogonality and normalization}

In this appendix, we will show that the combination of Jacobi polynomials given in (3.86) satisfies all the conditions that we demand. i.e., ( $i)$ They satisfy the recursion relation in (3.82) (which, of course, means the condition in (3.79) is satisfied) (ii) They are orthogonal to each other (3.80) with respect to the inner product defined in (3.78). We will also determine the normalization of the modes. 
Proof of recursion relation. The Jacobi Functions $P_{n}^{\alpha, \beta}(u)$ for special arguments $\alpha=$ $-\beta$ satisfy the following recursion relations (See eq. (2.34) and eq. (2.32) in [130])

$$
\begin{aligned}
\left(u(s+1)+n-\left(1-u^{2}\right) \partial_{u}\right) P_{s}^{n,-n}(u) & =(s+1) P_{s+1}^{n,-n}, \\
P_{s+1}^{n,-n}(u) & =\frac{2 s+1}{s+1} u P_{s}^{n,-n}(u)-\frac{\left(s^{2}-n^{2}\right)}{s(s+1)} P_{s-1}^{n,-n}(u) .
\end{aligned}
$$

Going to variables $u \equiv i \cot \sigma$ and defining $h_{s, n}(\sigma) \equiv \frac{e^{i n \sigma} P_{s-1}^{n,-n}(i \cot \sigma)}{\sin \sigma}$ and using the above two recursion relations we get

$$
-(s+1) h_{s+2}(\sigma)=i \frac{(2 s+1)}{s+1} \partial_{z} h_{s+1}(\sigma)+\frac{n^{2}-s^{2}}{s+1} h_{s, n}(\sigma) .
$$

Defining $H_{s, n}(\sigma) \equiv \frac{(s-1) !(s-1) !}{(2 s-3) !} e^{\frac{i \pi s}{2}} h_{s, n}(\sigma)$, the above equation can be seen to be

$$
H_{s+2, n}(\sigma)=\partial_{\sigma} H_{s+1, n}(\sigma)+\frac{s^{2}\left(n^{2}-s^{2}\right)}{4 s^{2}-1} H_{s, n}(\sigma) .
$$

If the above recursion is true for $H_{s, n}(\sigma)$, it is also true for $H_{s,-n}(\sigma)$. And therefore it also holds for the linear combination $g_{s, n}(\sigma)=H_{s, n}(\sigma)+H_{s,-n}(\sigma)$ and this completes the proof.

Orthogonality. To show that the functions $g_{s, n}(\sigma)$ is orthogonal, notice that it follows from the differential equation satisfied by Jacobi polynmial that the function $G_{s, n}(\sigma) \equiv$ $e^{i n \sigma} P_{s-1}^{n,-n}(\sigma)-e^{-i n \sigma} P_{s-1}^{-n, n}(\sigma)$ is a solution of a differential equation

$$
\left[n^{2}+\partial_{\sigma}^{2}-\frac{s(s-1)}{\sin ^{2} \sigma}\right] G_{s, n}(\sigma)=0 .
$$

Since $\int \frac{d \sigma}{\sin ^{2} \sigma} G_{s, n}^{*}(\sigma) G_{s^{\prime}, n^{\prime}}(\sigma) \propto\left\langle g_{s, n} \mid g_{s^{\prime}, n}\right\rangle$, we can plug the above relations into the equality

$$
\int d \sigma G_{s, n}^{*}(\chi, \sigma)\left(n^{2}+\partial_{\sigma}^{2}\right) G_{s^{\prime}, n^{\prime}}(\chi, \sigma)=\int d \chi d \sigma\left(n^{2}+\partial_{\sigma}^{2}\right) G_{s, n}^{*}(t, z) G_{s^{\prime}, n^{\prime}}(\chi, \sigma),
$$

to obtain $s^{\prime}\left(s^{\prime}-1\right)\left\langle g_{s, n} \mid g_{s^{\prime}, n^{\prime}}\right\rangle=s(s-1)\left\langle g_{s, n} \mid g_{s^{\prime}, n^{\prime}}\right\rangle$. Hence, we have

$$
\left\langle g_{s, n} \mid g_{s^{\prime}, n^{\prime}}\right\rangle=0 \quad \forall s \neq s^{\prime} .
$$

Normalization. Finally, we can now determine the normalization of the modes $N_{s, n} \equiv$ $\left\langle g_{s, n} \mid g_{s, n}\right\rangle$ in a purely algebraic way. Noting that

$$
\begin{aligned}
0 & \equiv\left\langle g_{s+2, n} \mid g_{s, n}\right\rangle=\left\langle\partial_{\sigma} g_{s+1, n}+F_{s, n} g_{s, n} \mid g_{s, n}\right\rangle=-\left\langle g_{s+1, n} \mid \partial_{\sigma} g_{s, n}\right\rangle+F_{s, n} N_{s, n} \\
& =-\left\langle g_{s+1, n} \mid g_{s+1, n}-F_{s-1, n} g_{s-1, n}\right\rangle+F_{s, n} N_{s, n} \\
& =N_{s, n} F_{s, n}-N_{s+1, n} .
\end{aligned}
$$

This gives

$$
\begin{aligned}
N_{s, n} & =N_{s-1, n} F_{s-1, n}=N_{s-2, n} F_{s-2, n} F_{s-1, n}=\ldots \\
& =n \pi(2 s-1)\left[\frac{(s-1) !}{(2 s-1) ! !}\right] \prod_{j=1}^{2-1}\left(n^{2}-j^{2}\right),
\end{aligned}
$$

where we used $N_{1, n}=n \pi$. 


\section{Lyapunov exponent from spin- $s$ soft mode}

In this appendix, we will estimate the contribution of spin- $s$ soft mode to the OTOC based on the soft mode eigenfunctions in appendix B. For simplicity, we do not consider the non-trivial contribution from non-contractible cycle. ${ }^{21}$

Recall that the essence of the long time limit of OTOCs lies in the following formula:

$$
\mathcal{F}_{s} \sim \sum_{|n| \geqq s} \frac{1}{\prod_{j=0}^{s-1}\left(n^{2}-j^{2}\right)} f_{s, n}(\chi, \sigma) f_{s,-n}\left(\chi^{\prime}, \sigma^{\prime}\right),
$$

where $f_{s, n}(\chi, \sigma)$ is the soft mode eigenfunction discussed in appendix B

$$
f_{s, n}(\chi, \sigma)=e^{-i n \chi} g_{s, n}(z)
$$

and

$$
g_{s, n}(\sigma)=0 \quad(|n|=0,1, \cdots, s-1) .
$$

In the calculation of OTOCs, it is useful to choose a special configuration. For this, we evaluate the soft mode eigenfunction at $z_{1}=\chi$ and $z_{2}=\chi+\pi$, or equivalently ${ }^{22}$ at $\sigma=-\frac{\pi}{2}$ :

$$
\begin{aligned}
& \frac{\delta_{\epsilon}\langle\phi(0) \bar{\phi}(\pi)\rangle}{\langle\phi(0) \bar{\phi}(\pi)\rangle} \\
& \quad=-\frac{w^{(s)}}{(s-1) !} \sum_{n} \epsilon_{n} e^{-i n \chi} \sum_{m=0}^{s-1} \frac{(n+s-1) !(s+m-1) !}{(n+m) ! m !(s-m-1) !}(-1)^{s+m} \frac{\left(e^{-i \frac{n \pi}{2}}+(-1)^{s} e^{i \frac{n \pi}{2}}\right)}{2^{m}} .
\end{aligned}
$$

For even $s$, this simply becomes

$$
\frac{\delta_{\epsilon}\left\langle\phi\left(\tau_{1}\right) \bar{\phi}\left(\tau_{2}\right)\right\rangle}{\left\langle\phi\left(\tau_{1}\right) \bar{\phi}\left(\tau_{2}\right)\right\rangle}=-\frac{w^{(s)}}{(s-1) !} \sum_{n} \epsilon_{n} e^{-i n \frac{t_{1}+t_{2}}{2}} \cos \left(\frac{n \pi}{2}\right) \prod_{m=1}^{\frac{s-1}{2}}\left[n^{2}-(2 m-1)^{2}\right],
$$

and, for odd $s$, we have

$$
\frac{\delta_{\epsilon}\left\langle\phi\left(\tau_{1}\right) \bar{\phi}\left(\tau_{2}\right)\right\rangle}{\left\langle\phi\left(\tau_{1}\right) \bar{\phi}\left(\tau_{2}\right)\right\rangle}=-i \frac{w^{(s)}}{(s-1) !} \sum_{n} \epsilon_{n} e^{-i n \frac{t_{1}+t_{2}}{2}} \sin \left(\frac{n \pi}{2}\right) n \prod_{m=2}^{\frac{s}{2}}\left[n^{2}-(2 m-2)^{2}\right] .
$$

As in section 4, we will evaluate the Euclidean four point function in (D.1) at the following points:

$$
\left(\chi, \sigma ; \chi^{\prime}, \sigma^{\prime}\right)=\left(\chi,-\frac{\pi}{2} ;-\frac{\pi}{2},-\frac{\pi}{2}\right) .
$$

One can easily see from (D.5) and (D.6) that $g_{s, n}\left(-\frac{\pi}{2}\right)$ vanishes at $n-s \equiv 1(\bmod 2)$ i.e.,

$$
g_{s, n}\left(-\frac{\pi}{2}\right)=0 \quad \text { for } \quad n= \pm(s+1), \pm(s+3), \pm(s+5), \cdots .
$$

\footnotetext{
${ }^{21}$ We guess that they would not change the Lyapunov exponent, but change the overall coefficient of exponential growth by order 1 .

${ }^{22}$ If we recover $\tau$, it corresponds to $\sigma=-\frac{\tau}{4}$.
} 
Furthermore, the other non-vanishing functions become

$$
g_{s, n}\left(-\frac{\pi}{2}\right) \sim\left\{\begin{array}{ll}
n\left(n^{2}-2^{2}\right)\left(n^{2}-4^{2}\right) \times \cdots \times\left[n^{2}-(s-2)^{2}\right] & \text { for } \quad s: \text { even } \\
\left(n^{2}-1\right)\left(n^{2}-3^{2}\right) \times \cdots \times\left[n^{2}-(s-2)^{2}\right] & \text { for } \quad s: \text { odd }
\end{array} .\right.
$$

Here, we omit the numerical coefficients which depend on spin $s$, but they will not have any influence on the Lyapunov exponent in our case. For even $s$, one can express the infinite series as a contour integral

$$
\mathcal{F}_{s}(\chi) \sim \frac{1}{2 \pi i} \oint_{\mathcal{C}} \frac{\frac{\pi}{2}}{\sin \frac{\pi \zeta}{2}} \frac{\left(\zeta^{2}-2^{2}\right) \times \cdots \times\left[\zeta^{2}-(s-2)^{2}\right]}{\left(\zeta^{2}-1\right) \times \cdots \times\left[\zeta^{2}-(s-1)^{2}\right]} e^{-i \zeta \chi}
$$

where $\mathcal{C}_{s}$ is a sum of counterclockwise circles centered at $\zeta= \pm s, \pm(s+2), \pm(s+4), \cdots$ with small radius. By deforming the contour, it can be changed into a contour integral along $\mathcal{C}^{\prime}$ which is a collection of clockwise circle centered at simple poles $\zeta=0, \pm 1, \pm 3, \cdots, \pm(s-1)$. Hence, evaluating the residues, we have

$$
\mathcal{F}_{s}(\chi) \sim \sum_{\substack{m=0 \\ n=-s+1+2 m}}^{s-1} a_{s, n} e^{-i n \chi}
$$

where $a_{s, n}$ is a constant of order $\mathcal{O}(1 / c)$. For real time OTOC, we take the analytic continuation

$$
\chi=\frac{2 \pi}{\tau}\left(\phi+i \frac{t_{E}}{l}\right) \Longrightarrow \frac{2 \pi}{i \beta}(l \phi-t) .
$$

Here, we retrieve the modular parameter $\tau=\frac{i \beta}{l}$ for non-rotating BTZ black hole. Then, the contribution of spin- $s$ field to OTOC is found to be

$$
\mathcal{F}_{s}(t) \sim \sum_{\substack{m=0 \\ n=-s+1+2 m}}^{s-1} a_{s, n} e^{-\frac{2 \pi}{\beta} n(l \phi-t)}=a_{s, s-1} e^{\frac{2 \pi}{\beta}(s-1)(t-l \phi)}+a_{s, s-3} e^{\frac{2 \pi}{\beta}(s-2)(t-l \phi)}+\cdots
$$

Then, one can easily read off the leading exponential growth, and the Lyapunov exponent is

$$
\lambda_{s}=\frac{2 \pi(s-1)}{\beta} .
$$

Also, one can repeat the same analysis for odd $s$, and one can obtain a similar result. That is, the OTOCs will be written as follow.

$$
\mathcal{F}_{s}(\chi) \sim \frac{1}{2 \pi i} \oint_{\mathcal{C}^{\prime}} \frac{\frac{\pi}{2}}{\cos \frac{\pi \zeta}{2}} \frac{\left(\zeta^{2}-1\right) \times \cdots \times\left[\zeta^{2}-(s-2)^{2}\right]}{\zeta^{2}\left(\zeta^{2}-2^{2}\right) \times \cdots \times\left[\zeta^{2}-(s-1)^{2}\right]} e^{-i \zeta \tau},
$$

where the deformed contour $\mathcal{C}^{\prime}$ is a collection of clockwise circle centered at $s=$ $0, \pm 2, \cdots, \pm(s-1)$. Note that in contrast to the previous even $s$, the integrand for odd $s$ has double pole at $\zeta=0$, and this would give extra contribution to the OTOCs. After 
analytic continuation to real time, we have

$$
\begin{aligned}
\mathcal{F}_{s} & \sim \sum_{\substack{m=0 \\
n=-s+1+2 m}}^{\frac{s-3}{2}} a_{s, n} e^{-\frac{2 \pi}{\beta} n(l \phi-t)}+a_{s, 0} \frac{2 \pi}{\beta}(t-l \phi)+\sum_{\substack{m=\frac{s+1}{2} \\
n=-s+1+2 m}}^{s-1} a_{s, n} e^{-\frac{2 \pi}{\beta} n(l \phi-t)} \\
& =a_{s, s-1} e^{\frac{2 \pi}{\beta}(s-1)(t-l \phi)}+a_{s, s-2} e^{\frac{2 \pi}{\beta}(s-2)(t-l \phi)}+\cdots+a_{s, 0} \frac{2 \pi}{\beta}(t-l \phi)+\cdots
\end{aligned}
$$

Note that the linear growth comes from the double pole at $\zeta=0$. The Lyapunov exponent is as before

$$
\lambda_{s}=\frac{2 \pi(s-1)}{\beta} .
$$

But, for $s=0$, there is no exponential growth, and therefore, the Lyapunov exponent is zero, but the OTOC grows linearly in time $[13,131]$.

\section{E OTOC in $2 D$ higher spin gravity}

In 2D gravity, $\mathrm{BF}$ theory with $\mathrm{SL}(N, \mathbb{R})$ can describe 2D higher spin gravity $[94,132,133]$, which is analogous to the three-dimensional Chern-Simons higher spin theory. Unlike Chern-Simon gravity, BF theory has only one $\mathrm{SL}(N, \mathbb{R})$ gauge connection, but it has extra player, $\operatorname{sl}(N)$ Dilaton field $\Phi$. The action of BF theory is given by

$$
S_{\mathrm{BF}}=\int \operatorname{tr}[\Phi(d A+A \wedge A)]
$$

where $A$ and $\Phi$ is gauge connection and dilaton field belonging to $\operatorname{sl}(N)$. The equation of motion is given by

$$
d A+A \wedge A=0, \quad d \Phi+[A, \Phi]=0 .
$$

One can fix a gauge [94] as in the 3D Chern-Simons gravity:

$$
A=b^{-1}(r)(d+a(\tau) d \tau) b(r)
$$

where we define

$$
b \equiv e^{r L_{0}}
$$

Like the asymptotic AdS condition for 3D, one can write the asymptotic AdS condition as

$$
a=L_{1}(-1)^{s} \sum_{s=2}^{N} \mathcal{W}_{s}(\tau) W_{-s+1}^{(s)} .
$$

In BF theory, we are also interested in a fixed constant background $a_{\beta}$ and fluctuations around it. i.e.,

$$
a=h^{-1}(\tau)\left(a_{\beta}+\partial_{\tau}\right) h(\tau)
$$

where $h(\tau)$ is a smooth gauge transformation connected to identity. Since the story about the gauge connection is almost the same as that of Chern-Simons gravity, let us focus on 
the dilaton field. The equation of motion of dilton with constant background $a_{\beta}$ is given by

$$
\partial_{\tau} \varphi+\left[a_{\beta}, \varphi\right]=0,
$$

and, its solution is easily found to be

$$
\varphi=e^{-a_{\beta} t} c e^{a_{\beta} t},
$$

where $c$ is a constant $\operatorname{sl}(N)$ matrix. Now, we consider the dilaton with a non-constant background $a=h^{-1}\left(a_{\beta}+\partial_{\tau}\right) h$ which is connected to the constant solution $a_{\beta}$ by gauge transformation $h$ :

$$
\partial_{\tau} \phi+[a, \phi]=0 .
$$

It is also easy to show that the solution is given by

$$
\phi=h^{-1} \varphi h=h^{-1} e^{-a_{\beta} \tau} c e^{a_{\beta} \tau} h,
$$

where $c$ is again a constant $\operatorname{sl}(N)$ matrix. Note that the solution of the dilaton can be thought as infinitesimal isometry of $\mathrm{AdS}_{2}$, and this can be easily seen from the equation of motion for the dilaton.

The dilaton solutions (or, infinitesimal isometries) are related by similarity transformation if and only if they have identical Casimirs $\mathcal{C}_{n}$. Here, we define $n_{\text {th }}$ order Casimir of the dilaton solution $\phi$ by

$$
\mathcal{C}_{n} \equiv-\frac{1}{n} \operatorname{tr}\left(\phi^{n}\right)
$$

On the other hand, for a given dilaton solution $\phi$ in (E.10), one can find upper-triangular matrix $B$ such that [86]

$$
B^{-1} c B=\left(\begin{array}{cccccc}
0 & u_{2} & u_{3} & \cdots & u_{N-1} & u_{N} \\
1 & 0 & 0 & \cdots & 0 & 0 \\
0 & 1 & 0 & \cdots & 0 & 0 \\
\vdots & & & \cdots & \vdots & \vdots \\
0 & 0 & 0 & \cdots & 0 & 0 \\
0 & 0 & 0 & \cdots & 1 & 0
\end{array}\right) \equiv K
$$

where $u_{i}$ 's (or, the matrix $K$ ) are defined via

$$
\operatorname{tr} \phi^{n}=\operatorname{tr} c^{n}=\operatorname{tr} K^{n} \quad(n=2,3, \cdots, N) .
$$

Here, by rescaling $B$, one can consider $B$ as constant $\operatorname{SL}(N)$ matrix. Hence, for a given connection $a$, two dilaton solutions with identical Casimirs can be related to the same matrix $K$ by similarity transformation, and therefore they are also related by similarity transformation. i.e.,

$$
\phi^{\prime}=v^{-1} \phi v \quad \text { where } \quad v=h^{-1} e^{-a_{\beta} \tau} c^{\prime} e^{a_{\beta} \tau} h,
$$

where $c^{\prime}$ is a constant $\operatorname{SL}(N)$ matrix. Now, for a given $u_{j}(j=2,3, \cdots, N)$ in (E.12), or equivalently for given eigenvalues $\mu_{j}$ of the matrix $K(j=1,2, \cdots, N)$, we will consider a 
constant dilaton solution in the $a_{\beta}$ background. Such a constant dilaton solution should commute with $a_{\beta}$ by the equation of motion:

$$
\left[a_{\beta}, \psi\right]=0 .
$$

Therefore, one can simultaneously diagonalize $a_{\beta}$ and $\psi$. One can show that $\psi$ is a polynomial of $a_{\beta}$ of which coefficients are made of Casimirs and the eigenvalues of $a_{\beta}$ which corresponds to the holonomy. Since $\psi$ is $N \times N$ matrix, it is enough to consider the following polynomial.

$$
\psi=\sum_{j=0}^{N-1} q_{j} a_{\beta}^{n} \quad \text { and } \quad \operatorname{tr}[\psi]=0,
$$

and, we will determine the coefficient $q_{j}$ 's. Since one can simultaneously diagonalize $\psi$ and $a_{\beta}$, (E.16) can be written as follow.

$$
\left(\begin{array}{llll}
q_{0} & q_{1} & \cdots & q_{N-1}
\end{array}\right)\left(\begin{array}{cccc}
1 & 1 & \cdots & 1 \\
\lambda_{1} & \lambda_{2} & \cdots & \lambda_{N} \\
\lambda_{1}^{2} & \lambda_{2}^{2} & \cdots & \lambda_{N}^{2} \\
\vdots & \vdots & & \vdots \\
\lambda_{1}^{N-1} & \lambda_{2}^{N-1} & \cdots & \lambda_{N}^{N-1}
\end{array}\right)=\left(\begin{array}{llll}
\mu_{1} & \mu_{2} & \cdots & \mu_{N}
\end{array}\right)
$$

where $\lambda_{j}$ 's are eigenvalues of $a_{\beta}$ and $\mu_{j}$ 's are the given eigenvalue of $\psi$. By inverting the matrix $(M)_{i j} \equiv \lambda_{i}^{j}$, one can determine $q_{j}$ 's in terms of $\lambda$ 's and $\mu$ 's.

For a general dilaton solution in a non-constant connection $a$, the asymptotic AdS solution that we are interested in is written as

$$
\begin{aligned}
a & =h^{-1}\left(a_{\mathrm{BTZ}}+\partial_{\tau}\right) h, \\
\phi(\tau) & =h^{-1}(\tau) v^{-1}(\tau) \psi v(\tau) h(\tau),
\end{aligned}
$$

where $v$ is the isometry of the constant background $a_{\beta}$ in (E.8). Then, using the above argument, one can express $\psi$ in terms of $a_{\beta}$ as follow.

$$
\psi=\sum_{j=0}^{N-1} q_{j} a_{\beta}^{j},
$$

where $q_{j}$ is a constant depending on Casimir of the dilaton and holonomy of the connection. Hence, we have

$$
\phi(\tau)=\sum_{j=0}^{N-1} q_{j}\left[a-\tilde{h}^{-1} \partial_{\tau} \tilde{h}\right]^{j},
$$

where we defined $\tilde{h} \equiv v h$. For these solutions, we need to add appropriate boundary condition to lead a consistent variational principle with suitable boundary condition. Unlike $\mathrm{AdS}_{3}$, it is not easy to find such a boundary term and boundary condition which is consistent with (E.18), (E.19) and (E.21). If there exists such a boundary term and a boundary condition, the reasonable guess for the on-shell action might be written as

$$
S_{\text {on-shell }} \stackrel{?}{=} \int d \tau \mathcal{C}_{2} \mathcal{W}^{(2)}(\tau)
$$


where $\mathcal{C}_{s}$ is $s_{\text {th }}$ order Casimir. If we consider a dilaton solution of which $\mathcal{C}_{2}$ is the only non-zero Casimir. i.e., $C_{s}=0$ for $(s>2)$. For such a solution, the on-shell action can be written as

$$
S_{\text {on-shell }} \stackrel{?}{=} \int d \tau \sum_{s=2}^{N} \mathcal{C}_{s} \mathcal{W}^{(s)}(\tau) .
$$

This would be too naive guess. However, it might be to guess that the quadratic on-shell action would be

$$
S_{\text {on-shell }}=c \sum_{s=2}^{N} \prod_{j=0}^{s-1}\left(n^{2}-j^{2}\right) \zeta_{-n}^{(s)} \zeta_{n}^{(s)} .
$$

Here, we assume that the background has $s l(N)$ isometry, and we demand that the on-shell action vanishes for the $s l(N)$ isometry. Then, the two point function of spin-s soft mode can be read off as

$$
\left\langle\zeta_{n}^{(s)} \zeta_{-n}^{(s)}\right\rangle \sim \frac{1}{c} \frac{1}{\prod_{j=0}^{s-1}\left(n^{2}-j^{2}\right)} .
$$

To evaluate the OTOC in 2D higher spin gravity, one can consider a Wilson line ${ }^{23}$ between $\left(r_{1}, \tau_{1}\right)$ and $\left(r_{2}, \tau_{2}\right)$ :

$$
W\left(r_{1}, \tau_{1} ; r_{2}, \tau_{2}\right) \equiv \mathcal{P} \exp \left[-\int_{\left(r_{2}, \tau_{2}\right)}^{\left(r_{1}, \tau_{1}\right)} A\right]=b^{-1}(r) e^{-a_{\beta}\left(\tau_{1}-\tau_{2}\right)} b\left(r_{2}\right)
$$

And, we define our bi-local field by a particular component of Wilson line:

$$
\Phi\left(\tau_{1}, \tau_{2}\right) \equiv \lim _{r \rightarrow \infty}\left[e^{2 h r} W\left(r, \tau_{1} ; r, \tau_{2}\right)\right]_{N, 1},
$$

where the conformal dimension $h$ is given by $h=-\frac{1}{2}(N-1)$ like $\mathrm{AdS}_{3}$ case. In the nonconstant background in (E.18), the gravitationally dressed bi-local field can be written as

$$
\begin{aligned}
\Phi^{\mathrm{dressed}}\left(\tau_{1}, \tau_{2}\right) & \equiv \lim _{r \rightarrow \infty}\left[e^{2 h r} \mathcal{P} \exp \left(-\int_{\left(r_{2}, \tau_{2}\right)}^{\left(r_{1}, \tau_{1}\right)} A\right)\right]_{N, 1} \\
& =\lim _{r \rightarrow \infty}\left[e^{2 h r} b^{-1}(r) h^{-1}\left(\tau_{1}\right) e^{-a_{\beta}\left(\tau_{1}-\tau_{2}\right)} h\left(\tau_{2}\right) b(r)\right]_{N, 1}
\end{aligned}
$$

Then, as in $\mathrm{AdS}_{3}$ case, the soft mode expansion of the dressed bi-local field allows us to evaluate OTOCs, which is exactly the same as the OTOTC calculation in appendix D.

\section{F Higher spin Schwarzian and connections to Toda theory}

In section 2.2 we only worked to quadratic order in fluctuations. In this appendix we will work out the results for finite fluctuations. For technical reasons we were able to carry out the calculations only at zero temperature and vanishing higher spin charges. The calculation which follows is based on [93], but such a form has been observed for a long time in various literature [91, 92, 94, 95].

\footnotetext{
${ }^{23}$ See $[54,116,117]$ for OTOC calculation from Wilson line for Schwarzian theory.
} 
Parametrizing the gauge connection $a_{z}$ by

$$
a_{z}(z)=L_{1}+\frac{1}{4} \mathcal{T}_{(2)}(z) L_{-1}-\frac{1}{4} \mathcal{T}_{(3)}(z) W_{-2}
$$

To compare with the main text, we have to use $\mathcal{T}_{(2)}=-\frac{8 \pi}{\kappa_{c s}} \mathcal{L}, \mathcal{T}_{(3)}=-\frac{2 \pi}{\kappa_{c s}} \mathcal{W}$. The transformation is given by

$$
a_{z}(z) \rightarrow \tilde{a}(z) \equiv g(z)^{-1}\left(\partial_{z}+a(z)\right) \gamma(z)=L_{1}+\frac{1}{4} \tilde{\mathcal{T}}_{(2)}(z) L_{-1}-\frac{1}{4} \tilde{\mathcal{T}}_{(3)}(z) W_{-2} .
$$

As mentioned in the beginning, we were able to calculate the finite transformations only for the cases when all the initial charges vanish, i.e $\mathcal{T}_{(2)}=\mathcal{T}_{(3)}=0$, i.e $a_{z}(z)=L_{1}$. Notice that $\tilde{\mathcal{T}}_{(2)}(z), \tilde{\mathcal{T}}_{(3)}(z)$ we read off from (F.2) is the analogue of the schwarzians for the $s l(3)$ case. First note that we can simplify the equations somewhat if we define $g(z)=e^{-z L_{1}} \tilde{g}(z)$. The equation we need to solve for then becomes

$$
\tilde{g}^{-1}(z) \partial_{z} \tilde{g}(z)=L_{1}+\frac{1}{4} \tilde{\mathcal{T}}_{(2)}(z) L_{-1}-\frac{1}{4} \tilde{\mathcal{T}}_{(3)}(z) W_{-2}
$$

We will decompose (the normalizations for the fields are chosen so that the equations turn out to be simple)

$$
\tilde{g}(z)=e^{\frac{1}{4} w_{2}(z) W_{2}-\sqrt{2} e_{+}(z) E_{+}-\sqrt{2} f_{+}(z) F_{+}} e^{-\phi_{1}(z) H_{1}-\phi_{2}(z) H_{2}} e^{\frac{1}{4} w_{-2}(z) W_{-2}-\frac{e_{-}(z) E_{-}}{\sqrt{2}}-\frac{f_{-}(z) F_{-}}{\sqrt{2}}},
$$

where the $s l(3)$ generators are chosen in chevalley basis

$$
\begin{array}{ll}
H_{1}=\left(\begin{array}{ccc}
1 & 0 & 0 \\
0 & -1 & 0 \\
0 & 0 & 0
\end{array}\right), H_{2}=\left(\begin{array}{ccc}
0 & 0 & 0 \\
0 & 1 & 0 \\
0 & 0 & -1
\end{array}\right), E_{+}=\left(\begin{array}{lll}
0 & 0 & 0 \\
1 & 0 & 0 \\
0 & 0 & 0
\end{array}\right), F_{+}=\left(\begin{array}{lll}
0 & 0 & 0 \\
0 & 0 & 0 \\
0 & 1 & 0
\end{array}\right) \\
E_{-}=\left(\begin{array}{lll}
0 & 1 & 0 \\
0 & 0 & 0 \\
0 & 0 & 0
\end{array}\right), & F_{-}=\left(\begin{array}{lll}
0 & 0 & 0 \\
0 & 0 & 1 \\
0 & 0 & 0
\end{array}\right) .
\end{array}
$$

We will not need the explicit relation between the Chevally basis and standard basis given in (A.1) (A.3). Demanding (F.3) gives

$$
\begin{aligned}
e_{+}^{\prime} & =-e^{2 \phi_{1}-\phi_{2}}, & e_{-} & =\phi_{1}^{\prime}, \\
f_{+}^{\prime} & =-e^{2 \phi_{2}-\phi_{1}}, & f_{-} & =\phi_{2}^{\prime}, \\
w_{2}^{\prime} & =f_{+} \partial_{+} e_{+}^{\prime}-e_{+} \partial_{+} f_{+}, & 4 w_{-2} & =f_{-}^{\prime}-e_{-}^{\prime}+\left(\phi_{1}^{\prime}\right)^{2}-\left(\phi_{2}^{\prime}\right)^{2} .
\end{aligned}
$$

We can solve for $f_{ \pm}, e_{ \pm}, w_{ \pm}$in terms of $\phi_{1}, \phi_{2}$ and finally obtain

$$
\begin{aligned}
\tilde{\mathcal{T}}_{2} & =\left\{\phi_{1}^{\prime \prime}-\left(\phi_{1}^{\prime}\right)^{2}+\frac{1}{2} \phi_{1}^{\prime} \phi_{2}^{\prime}\right\}+\phi_{1} \leftrightarrow \phi_{2}, \\
& =\left\{\frac{e_{+}^{\prime \prime \prime}}{e_{+}^{\prime}}-\frac{4}{3}\left(\frac{e_{+}^{\prime \prime}}{e_{+}^{\prime}}\right)^{2}-\frac{1}{6} \frac{e_{+}^{\prime \prime}}{e_{+}^{\prime}} \frac{f_{+}^{\prime \prime}}{f_{+}^{\prime}}\right\}+e_{+} \leftrightarrow f_{+}, \\
2 \tilde{\mathcal{T}}_{3} & =\left\{\frac{1}{2} \phi_{1}^{\prime \prime \prime}-\frac{1}{2} \phi_{1}^{\prime \prime} \phi_{2}^{\prime}-\phi_{1}^{\prime \prime} \phi_{1}^{\prime}+\phi_{1}^{\prime 2} \phi_{2}^{\prime}\right\}-\phi_{1} \leftrightarrow \phi_{2}, \\
& =\left\{\frac{1}{6}\left[\frac{e_{+}^{\prime \prime \prime \prime}}{e_{+}^{\prime}}-5 \frac{e_{+}^{\prime \prime \prime}}{e_{+}^{\prime}} \frac{e_{+}^{\prime \prime}}{e_{+}^{\prime}}+\frac{40}{9}\left(\frac{e_{+}^{\prime \prime}}{e_{+}^{\prime}}\right)^{3}\right]-\frac{1}{6} \frac{e_{+}^{\prime \prime \prime}}{e_{+}^{\prime}} \frac{f_{+}^{\prime \prime}}{f_{+}^{\prime}}+\frac{5}{18} \frac{e_{+}^{\prime \prime}}{e_{+}^{\prime}}\left(\frac{f_{+}^{\prime \prime}}{f_{+}^{\prime}}\right)^{2}\right\}-e_{+} \leftrightarrow f_{+} .
\end{aligned}
$$


It is obvious that for the case of pure diffeomorphism we must set $e_{+}=f_{+}$or equivalently $\phi_{1}=\phi_{2}$. At this point, we can connect the above discussion to Toda field theory. The expression for $\mathcal{T}_{(2)}, \mathcal{T}_{(3)}$ in terms of $\phi_{1}, \phi_{2}$ are exactly same as that charges of Toda field theory (see eq. (3.47) of [93]).

Open Access. This article is distributed under the terms of the Creative Commons Attribution License (CC-BY 4.0), which permits any use, distribution and reproduction in any medium, provided the original author(s) and source are credited.

\section{References}

[1] S.H. Shenker and D. Stanford, Black holes and the butterfly effect, JHEP 03 (2014) 067 [arXiv:1306.0622] [INSPIRE].

[2] D.A. Roberts, D. Stanford and L. Susskind, Localized shocks, JHEP 03 (2015) 051 [arXiv: 1409.8180] [INSPIRE].

[3] S.H. Shenker and D. Stanford, Stringy effects in scrambling, JHEP 05 (2015) 132 [arXiv:1412.6087] [INSPIRE].

[4] S.H. Shenker and D. Stanford, Multiple shocks, JHEP 12 (2014) 046 [arXiv:1312.3296] [INSPIRE].

[5] J. Maldacena, S.H. Shenker and D. Stanford, A bound on chaos, JHEP 08 (2016) 106 [arXiv: 1503.01409] [INSPIRE].

[6] Y. Sekino and L. Susskind, Fast scramblers, JHEP 10 (2008) 065 [arXiv:0808. 2096] [INSPIRE].

[7] V. Jahnke, K.-Y. Kim and J. Yoon, On the chaos bound in rotating black holes, JHEP 05 (2019) 037 [arXiv: 1903.09086] [INSPIRE].

[8] A.L. Fitzpatrick and J. Kaplan, A quantum correction to chaos, JHEP 05 (2016) 070 [arXiv: 1601.06164] [INSPIRE].

[9] H. Chen, A.L. Fitzpatrick, J. Kaplan, D. Li and J. Wang, Degenerate operators and the $1 / c$ expansion: Lorentzian resummations, high order computations and super-Virasoro blocks, JHEP 03 (2017) 167 [arXiv:1606.02659] [INSPIRE].

[10] E. Perlmutter, Bounding the space of holographic CFTs with chaos, JHEP 10 (2016) 069 [arXiv: 1602.08272] [INSPIRE].

[11] J. Maldacena, D. Stanford and Z. Yang, Conformal symmetry and its breaking in two dimensional nearly anti-de-Sitter space, PTEP 2016 (2016) 12C104 [arXiv:1606.01857] [INSPIRE].

[12] P. Narayan and J. Yoon, SYK-like tensor models on the lattice, JHEP 08 (2017) 083 [arXiv: 1705.01554] [INSPIRE].

[13] J. Yoon, SYK models and SYK-like tensor models with global symmetry, JHEP 10 (2017) 183 [arXiv: 1707.01740] [INSPIRE].

[14] P. Narayan and J. Yoon, Supersymmetric SYK model with global symmetry, JHEP 08 (2018) 159 [arXiv:1712.02647] [INSPIRE].

[15] A. Gaikwad, L.K. Joshi, G. Mandal and S.R. Wadia, Holographic dual to charged SYK from $3 D$ gravity and Chern-Simons, arXiv:1802.07746 [INSPIRE]. 
[16] R.R. Poojary, BTZ dynamics and chaos, arXiv:1812.10073 [INSPIRE].

[17] S. Sachdev and J. Ye, Gapless spin fluid ground state in a random, quantum Heisenberg magnet, Phys. Rev. Lett. 70 (1993) 3339 [cond-mat/9212030] [INSPIRE].

[18] J. Polchinski and V. Rosenhaus, The spectrum in the Sachdev-Ye-Kitaev model, JHEP 04 (2016) 001 [arXiv: 1601.06768] [inSPIRE].

[19] A. Jevicki, K. Suzuki and J. Yoon, Bi-local holography in the SYK model, JHEP 07 (2016) 007 [arXiv: 1603.06246] [INSPIRE].

[20] J. Maldacena and D. Stanford, Remarks on the Sachdev-Ye-Kitaev model, Phys. Rev. D 94 (2016) 106002 [arXiv: 1604.07818] [inSPIRE].

[21] A. Jevicki and K. Suzuki, Bi-local holography in the SYK model: perturbations, JHEP 11 (2016) 046 [arXiv: 1608.07567] [INSPIRE].

[22] D.J. Gross and V. Rosenhaus, A generalization of Sachdev-Ye-Kitaev, JHEP 02 (2017) 093 [arXiv: 1610.01569] [INSPIRE].

[23] W. Fu, D. Gaiotto, J. Maldacena and S. Sachdev, Supersymmetric Sachdev-Ye-Kitaev models, Phys. Rev. D 95 (2017) 026009 [Addendum ibid. D 95 (2017) 069904] [arXiv: 1610.08917$]$ [INSPIRE].

[24] R. Gurau, The 1/N expansion of colored tensor models, Annales Henri Poincaré 12 (2011) 829 [arXiv: 1011.2726] [INSPIRE].

[25] S. Carrozza and A. Tanasa, $O(N)$ random tensor models, Lett. Math. Phys. 106 (2016) 1531 [arXiv: 1512.06718] [INSPIRE].

[26] E. Witten, An SYK-like model without disorder, arXiv:1610.09758 [INSPIRE].

[27] R. Gurau, The complete $1 / N$ expansion of a SYK-like tensor model, Nucl. Phys. B 916 (2017) 386 [arXiv: 1611.04032] [INSPIRE].

[28] I.R. Klebanov and G. Tarnopolsky, Uncolored random tensors, melon diagrams and the Sachdev-Ye-Kitaev models, Phys. Rev. D 95 (2017) 046004 [arXiv:1611.08915] [inSPIRE].

[29] G. Sárosi, AdS $S_{2}$ holography and the SYK model, PoS (Modave2017)001 (2018) [arXiv: 1711.08482] [INSPIRE].

[30] V. Jahnke, Recent developments in the holographic description of quantum chaos, Adv. High Energy Phys. 2019 (2019) 9632708 [arXiv: 1811.06949] [InSPIRE].

[31] S.R. Das, A. Jevicki and K. Suzuki, Three dimensional view of the SYK/AdS duality, JHEP 09 (2017) 017 [arXiv: 1704.07208] [INSPIRE].

[32] S. Chaudhuri, V.I. Giraldo-Rivera, A. Joseph, R. Loganayagam and J. Yoon, Abelian tensor models on the lattice, Phys. Rev. D 97 (2018) 086007 [arXiv:1705.01930] [INSPIRE].

[33] J. Yoon, Supersymmetric SYK model: bi-local collective superfield/supermatrix formulation, JHEP 10 (2017) 172 [arXiv:1706.05914] [INSPIRE].

[34] C. Peng, M. Spradlin and A. Volovich, Correlators in the $N=2$ supersymmetric SYK model, JHEP 10 (2017) 202 [arXiv:1706.06078] [INSPIRE].

[35] K. Bulycheva, A note on the SYK model with complex fermions, JHEP 12 (2017) 069 [arXiv: 1706.07411] [INSPIRE].

[36] C. Krishnan, S. Sanyal and P.N. Bala Subramanian, Quantum chaos and holographic tensor models, JHEP 03 (2017) 056 [arXiv:1612.06330] [InSPIRE]. 
[37] C. Krishnan, K.V.P. Kumar and S. Sanyal, Random matrices and holographic tensor models, JHEP 06 (2017) 036 [arXiv:1703.08155] [INSPIRE].

[38] R. de Mello Koch, R. Mello Koch, D. Gossman and L. Tribelhorn, Gauge invariants, correlators and holography in bosonic and fermionic tensor models, JHEP 09 (2017) 011 [arXiv: 1707.01455] [INSPIRE].

[39] M.M. Qaemmaqami, Butterfly effect in 3D gravity, Phys. Rev. D 96 (2017) 106012 [arXiv: 1707.00509] [INSPIRE].

[40] T. Azeyanagi, F. Ferrari and F.I. Schaposnik Massolo, Phase diagram of planar matrix quantum mechanics, tensor and Sachdev-Ye-Kitaev models, Phys. Rev. Lett. 120 (2018) 061602 [arXiv: 1707.03431] [INSPIRE].

[41] K. Bulycheva, I.R. Klebanov, A. Milekhin and G. Tarnopolsky, Spectra of operators in large $N$ tensor models, Phys. Rev. D 97 (2018) 026016 [arXiv: 1707.09347] [InSPIRE].

[42] C. Krishnan, K.V. Pavan Kumar and D. Rosa, Contrasting SYK-like models, JHEP 01 (2018) 064 [arXiv: 1709.06498] [INSPIRE].

[43] A. Kitaev and S.J. Suh, The soft mode in the Sachdev-Ye-Kitaev model and its gravity dual, JHEP 05 (2018) 183 [arXiv:1711.08467] [INSPIRE].

[44] S.R. Das, A. Ghosh, A. Jevicki and K. Suzuki, Space-time in the SYK model, JHEP 07 (2018) 184 [arXiv: 1712.02725] [inSPIRE].

[45] P. Diaz and S.-J. Rey, Invariant operators, orthogonal bases and correlators in general tensor models, Nucl. Phys. B 932 (2018) 254 [arXiv:1801.10506] [INSPIRE].

[46] S. Giombi, I.R. Klebanov, F. Popov, S. Prakash and G. Tarnopolsky, Prismatic large N models for bosonic tensors, Phys. Rev. D 98 (2018) 105005 [arXiv:1808.04344] [InSPIRE].

[47] J. Maldacena and X.-L. Qi, Eternal traversable wormhole, arXiv:1804.00491 [INSPIRE].

[48] C.-M. Chang, S. Colin-Ellerin and M. Rangamani, On melonic supertensor models, JHEP 10 (2018) 157 [arXiv: 1806.09903] [InSPIRE].

[49] M. Berkooz, P. Narayan and J. Simon, Chord diagrams, exact correlators in spin glasses and black hole bulk reconstruction, JHEP 08 (2018) 192 [arXiv: 1806.04380] [INSPIRE].

[50] D. Benedetti and R. Gurau, 2PI effective action for the SYK model and tensor field theories, JHEP 05 (2018) 156 [arXiv: 1802.05500] [INSPIRE].

[51] T. Nosaka, D. Rosa and J. Yoon, The Thouless time for mass-deformed SYK, JHEP 09 (2018) 041 [arXiv: 1804.09934] [INSPIRE].

[52] C. Peng, $N=(0,2)$ SYK, chaos and higher-spins, JHEP 12 (2018) 065 [arXiv: 1805.09325] [inSPIRE].

[53] C. Ahn and C. Peng, Chiral algebras of two-dimensional SYK models, arXiv:1812.05106 [INSPIRE].

[54] A. Blommaert, T.G. Mertens and H. Verschelde, The Schwarzian theory - a Wilson line perspective, JHEP 12 (2018) 022 [arXiv: 1806.07765] [INSPIRE].

[55] T.G. Mertens, The Schwarzian theory — origins, JHEP 05 (2018) 036 [arXiv: 1801.09605] [INSPIRE].

[56] F. Sun, Y. Yi-Xiang, J. Ye and W.M. Liu, Classification of the quantum chaos in colored Sachdev-Ye-Kitaev models, arXiv: 1903.02213 [INSPIRE]. 
[57] A.M. García-García, T. Nosaka, D. Rosa and J.J.M. Verbaarschot, Quantum chaos transition in a two-site SYK model dual to an eternal traversable wormhole, arXiv: 1901.06031 [INSPIRE].

[58] R. de Mello Koch, W. LiMing, H.J.R. Van Zyl and J.P. Rodrigues, Chaos in the fishnet, Phys. Lett. B 793 (2019) 169 [arXiv: 1902.06409] [InSPIRE].

[59] J. Kim, I.R. Klebanov, G. Tarnopolsky and W. Zhao, Symmetry breaking in coupled SYK or tensor models, Phys. Rev. X 9 (2019) 021043 [arXiv: 1902.02287] [INSPIRE].

[60] F. Ferrari and F.I. Schaposnik Massolo, Phases of melonic quantum mechanics, arXiv: 1903.06633 [INSPIRE].

[61] K. Jensen, Chaos in AdS 2 holography, Phys. Rev. Lett. 117 (2016) 111601 [arXiv: 1605. 06098] [INSPIRE].

[62] D. Bagrets, A. Altland and A. Kamenev, Sachdev-Ye-Kitaev model as Liouville quantum mechanics, Nucl. Phys. B 911 (2016) 191 [arXiv:1607.00694] [INSPIRE].

[63] D. Grumiller, R. McNees, J. Salzer, C. Valcárcel and D. Vassilevich, Menagerie of $A d S_{2}$ boundary conditions, JHEP 10 (2017) 203 [arXiv: 1708.08471] [INSPIRE].

[64] G. Mandal, P. Nayak and S.R. Wadia, Coadjoint orbit action of Virasoro group and two-dimensional quantum gravity dual to SYK/tensor models, JHEP 11 (2017) 046 [arXiv: 1702.04266] [INSPIRE].

[65] M. Cvetič and I. Papadimitriou, AdS 2 holographic dictionary, JHEP 12 (2016) 008 [Erratum ibid. 01 (2017) 120] [arXiv: 1608.07018] [INSPIRE].

[66] A. Castro, F. Larsen and I. Papadimitriou, $5 D$ rotating black holes and the $n A d S_{2} / n C F T_{1}$ correspondence, JHEP 10 (2018) 042 [arXiv:1807.06988] [INSPIRE].

[67] J. Cotler and K. Jensen, A theory of reparameterizations for AdS $S_{3}$ gravity, JHEP 02 (2019) 079 [arXiv: 1808.03263] [INSPIRE].

[68] A. Achucarro and P.K. Townsend, A Chern-Simons action for three-dimensional anti-de Sitter supergravity theories, Phys. Lett. B 180 (1986) 89 [INSPIRE].

[69] E. Witten, $(2+1)$-dimensional gravity as an exactly soluble system, Nucl. Phys. B 311 (1988) 46 [INSPIRE].

[70] M.P. Blencowe, A consistent interacting massless higher spin field theory in $D=(2+1)$, Class. Quant. Grav. 6 (1989) 443 [INSPIRE].

[71] A. Campoleoni, S. Fredenhagen, S. Pfenninger and S. Theisen, Asymptotic symmetries of three-dimensional gravity coupled to higher-spin fields, JHEP 11 (2010) 007 [arXiv: 1008.4744] [INSPIRE].

[72] A. Campoleoni, S. Fredenhagen and S. Pfenninger, Asymptotic W-symmetries in three-dimensional higher-spin gauge theories, JHEP 09 (2011) 113 [arXiv:1107.0290] [INSPIRE].

[73] C. Fronsdal, Massless fields with integer spin, Phys. Rev. D 18 (1978) 3624 [InSPIRE].

[74] J. Fang and C. Fronsdal, Massless fields with half integral spin, Phys. Rev. D 18 (1978) 3630 [INSPIRE].

[75] C. Fronsdal, Singletons and massless, integral spin fields on de Sitter space, Phys. Rev. D 20 (1979) 848 [INSPIRE]. 
[76] M.A. Vasiliev, Consistent equation for interacting gauge fields of all spins in (3+1)-dimensions, Phys. Lett. B 243 (1990) 378 [INSPIRE].

[77] M.A. Vasiliev, Higher spin gauge theories in four-dimensions, three-dimensions and two-dimensions, Int. J. Mod. Phys. D 5 (1996) 763 [hep-th/9611024] [INSPIRE].

[78] S.F. Prokushkin and M.A. Vasiliev, Higher spin gauge interactions for massive matter fields in 3D AdS space-time, Nucl. Phys. B 545 (1999) 385 [hep-th/9806236] [InSPIRE].

[79] S. Prokushkin and M.A. Vasiliev, 3D higher spin gauge theories with matter, in Theory of elementary particles. Proceedings, $31^{\text {st }}$ International Symposium Ahrenshoop, Buckow, Germany, 2-6 September 1997 [hep-th/9812242] [INSPIRE].

[80] X. Bekaert, S. Cnockaert, C. Iazeolla and M.A. Vasiliev, Nonlinear higher spin theories in various dimensions, in Higher spin gauge theories: proceedings, $1^{\text {st }}$ Solvay Workshop, Brussels, Belgium, 12-14 May 2004, pg. 132 [hep-th/0503128] [INSPIRE].

[81] M.R. Gaberdiel and R. Gopakumar, An AdS $S_{3}$ dual for minimal model CFTs, Phys. Rev. D 83 (2011) 066007 [arXiv: 1011.2986] [INSPIRE].

[82] I.R. Klebanov and A.M. Polyakov, AdS dual of the critical $O(N)$ vector model, Phys. Lett. B 550 (2002) 213 [hep-th/0210114] [INSPIRE].

[83] E. Sezgin and P. Sundell, Massless higher spins and holography, Nucl. Phys. B 644 (2002) 303 [Erratum ibid. B 660 (2003) 403] [hep-th/0205131] [INSPIRE].

[84] S. Giombi and X. Yin, Higher spin gauge theory and holography: the three-point functions, JHEP 09 (2010) 115 [arXiv:0912.3462] [INSPIRE].

[85] K. Papadodimas and S. Raju, Correlation functions in holographic minimal models, Nucl. Phys. B 856 (2012) 607 [arXiv:1108. 3077] [INSPIRE].

[86] E. Hijano, P. Kraus and E. Perlmutter, Matching four-point functions in higher spin $A d S_{3} / C F T_{2}, J H E P 05$ (2013) 163 [arXiv:1302.6113] [INSPIRE].

[87] R. de Mello Koch, A. Jevicki, J.P. Rodrigues and J. Yoon, Canonical formulation of $O(N)$ vector/higher spin correspondence, J. Phys. A 48 (2015) 105403 [arXiv:1408.4800] [INSPIRE].

[88] R. de Mello Koch, A. Jevicki, J.P. Rodrigues and J. Yoon, Holography as a gauge phenomenon in higher spin duality, JHEP 01 (2015) 055 [arXiv:1408.1255] [INSPIRE].

[89] A. Jevicki and J. Yoon, Bulk from bi-locals in thermo field CFT, JHEP 02 (2016) 090 [arXiv: 1503.08484] [INSPIRE].

[90] J.R. David, S. Khetrapal and S.P. Kumar, Local quenches and quantum chaos from higher spin perturbations, JHEP 10 (2017) 156 [arXiv:1707.07166] [INSPIRE].

[91] M. Bershadsky and H. Ooguri, Hidden SL(n) symmetry in conformal field theories, Commun. Math. Phys. 126 (1989) 49 [InSPIRE].

[92] A. Marshakov and A. Morozov, A note on $W_{3}$ algebra, Nucl. Phys. B 339 (1990) 79 [INSPIRE].

[93] W. Li and S. Theisen, Some aspects of holographic W-gravity, JHEP 08 (2015) 035 [arXiv: 1504.07799] [INSPIRE].

[94] H.A. González, D. Grumiller and J. Salzer, Towards a bulk description of higher spin SYK, JHEP 05 (2018) 083 [arXiv: 1802.01562] [INSPIRE]. 
[95] C.-T. Ma and H. Shu, SL(3) Chern-Simons higher spin and open Toda chain theories, arXiv: 1902.10279 [INSPIRE].

[96] J. de Boer and J.I. Jottar, Thermodynamics of higher spin black holes in $A d S_{3}$, JHEP 01 (2014) 023 [arXiv: 1302.0816] [INSPIRE].

[97] M. Gutperle and P. Kraus, Higher spin black holes, JHEP 05 (2011) 022 [arXiv:1103.4304] [INSPIRE].

[98] M. Ammon, M. Gutperle, P. Kraus and E. Perlmutter, Spacetime geometry in higher spin gravity, JHEP 10 (2011) 053 [arXiv: 1106 . 4788] [INSPIRE].

[99] A. Castro, E. Hijano, A. Lepage-Jutier and A. Maloney, Black holes and singularity resolution in higher spin gravity, JHEP 01 (2012) 031 [arXiv:1110.4117] [INSPIRE].

[100] M.R. Gaberdiel, T. Hartman and K. Jin, Higher spin black holes from CFT, JHEP 04 (2012) 103 [arXiv: 1203.0015] [INSPIRE].

[101] A. Castro, R. Gopakumar, M. Gutperle and J. Raeymaekers, Conical defects in higher spin theories, JHEP 02 (2012) 096 [arXiv:1111.3381] [INSPIRE].

[102] M. Henneaux and S.-J. Rey, Nonlinear $W_{\infty}$ as asymptotic symmetry of three-dimensional higher spin anti-de Sitter gravity, JHEP 12 (2010) 007 [arXiv:1008.4579] [INSPIRE].

[103] V.K. Dobrev, G. Mack, V.B. Petkova, S.G. Petrova and I.T. Todorov, Harmonic analysis on the n-dimensional Lorentz group and its application to conformal quantum field theory, Lect. Notes Phys. 63 (1977) 1 [INSPIRE].

[104] R. de Mello Koch, A. Jevicki, K. Suzuki and J. Yoon, AdS maps and diagrams of bi-local holography, JHEP 03 (2019) 133 [arXiv:1810.02332] [INSPIRE].

[105] M.R. Gaberdiel and R. Gopakumar, Triality in minimal model holography, JHEP 07 (2012) 127 [arXiv: 1205.2472] [INSPIRE].

[106] E. Perlmutter, T. Prochazka and J. Raeymaekers, The semiclassical limit of $W_{N}$ CFTs and Vasiliev theory, JHEP 05 (2013) 007 [arXiv: 1210.8452] [INSPIRE].

[107] M. Ammon, P. Kraus and E. Perlmutter, Scalar fields and three-point functions in D $=3$ higher spin gravity, JHEP 07 (2012) 113 [arXiv:1111.3926] [INSPIRE].

[108] M.R. Gaberdiel, R. Gopakumar, T. Hartman and S. Raju, Partition functions of holographic minimal models, JHEP 08 (2011) 077 [arXiv:1106.1897] [INSPIRE].

[109] M. Ammon, A. Castro and N. Iqbal, Wilson lines and entanglement entropy in higher spin gravity, JHEP 10 (2013) 110 [arXiv:1306.4338] [INSPIRE].

[110] J. de Boer and J.I. Jottar, Entanglement entropy and higher spin holography in $A d S_{3}$, JHEP 04 (2014) 089 [arXiv:1306.4347] [INSPIRE].

[111] A. Castro, S. Detournay, N. Iqbal and E. Perlmutter, Holographic entanglement entropy and gravitational anomalies, JHEP 07 (2014) 114 [arXiv:1405.2792] [INSPIRE].

[112] J. de Boer, A. Castro, E. Hijano, J.I. Jottar and P. Kraus, Higher spin entanglement and $W_{N}$ conformal blocks, JHEP 07 (2015) 168 [arXiv: 1412.7520] [INSPIRE].

[113] A. Castro, N. Iqbal and E. Llabrés, Wilson lines and Ishibashi states in $A d S_{3} / \mathrm{CFT}_{2}$, JHEP 09 (2018) 066 [arXiv: 1805.05398] [INSPIRE].

[114] S. Ryu and T. Takayanagi, Holographic derivation of entanglement entropy from AdS/CFT, Phys. Rev. Lett. 96 (2006) 181602 [hep-th/0603001] [INSPIRE]. 
[115] S. Ryu and T. Takayanagi, Aspects of holographic entanglement entropy, JHEP 08 (2006) 045 [hep-th/0605073] [INSPIRE].

[116] A. Blommaert, T.G. Mertens and H. Verschelde, Fine structure of Jackiw-Teitelboim quantum gravity, arXiv:1812.00918 [INSPIRE].

[117] A. Blommaert, T.G. Mertens and H. Verschelde, Clocks and rods in Jackiw-Teitelboim quantum gravity, arXiv: 1902.11194 [INSPIRE].

[118] E. Keski-Vakkuri, Bulk and boundary dynamics in BTZ black holes, Phys. Rev. D 59 (1999) 104001 [hep-th/9808037] [INSPIRE].

[119] P. Kraus, H. Ooguri and S. Shenker, Inside the horizon with AdS/CFT, Phys. Rev. D 67 (2003) 124022 [hep-th/0212277] [INSPIRE].

[120] C.-M. Chang and X. Yin, Correlators in $W_{N}$ minimal model revisited, JHEP 10 (2012) 050 [arXiv: 1112.5459] [INSPIRE].

[121] A. Jevicki and J. Yoon, Field theory of primaries in $W_{N}$ minimal models, JHEP 11 (2013) 060 [arXiv: 1302.3851] [INSPIRE].

[122] C.-M. Chang and X. Yin, A semilocal holographic minimal model, Phys. Rev. D 88 (2013) 106002 [arXiv: 1302.4420] [INSPIRE].

[123] J. Murugan, D. Stanford and E. Witten, More on supersymmetric and $2 d$ analogs of the SYK model, JHEP 08 (2017) 146 [arXiv: 1706. 05362] [INSPIRE].

[124] F.M. Haehl and M. Rozali, Effective field theory for chaotic CFTs, JHEP 10 (2018) 118 [arXiv: 1808.02898] [INSPIRE].

[125] R.A. Davison, W. Fu, A. Georges, Y. Gu, K. Jensen and S. Sachdev, Thermoelectric transport in disordered metals without quasiparticles: the Sachdev-Ye-Kitaev models and holography, Phys. Rev. B 95 (2017) 155131 [arXiv:1612.00849] [INSPIRE].

[126] S. Grozdanov, K. Schalm and V. Scopelliti, Black hole scrambling from hydrodynamics, Phys. Rev. Lett. 120 (2018) 231601 [arXiv:1710.00921] [INSPIRE].

[127] M. Blake, H. Lee and H. Liu, A quantum hydrodynamical description for scrambling and many-body chaos, JHEP 10 (2018) 127 [arXiv:1801.00010] [INSPIRE].

[128] M. Blake, R.A. Davison, S. Grozdanov and H. Liu, Many-body chaos and energy dynamics in holography, JHEP 10 (2018) 035 [arXiv:1809.01169] [INSPIRE].

[129] S. Grozdanov, On the connection between hydrodynamics and quantum chaos in holographic theories with stringy corrections, JHEP 01 (2019) 048 [arXiv: 1811.09641] [INSPIRE].

[130] A. Wünsche, Operator methods and SU $(1,1)$ symmetry in the theory of Jacobi and of ultraspherical polynomials, Adv. Pure Math. 07 (2017) 213.

[131] S. Choudhury, A. Dey, I. Halder, L. Janagal, S. Minwalla and R. Poojary, Notes on melonic $O(N)^{q-1}$ tensor models, JHEP 06 (2018) 094 [arXiv: 1707.09352] [INSPIRE].

[132] M.A. Vasiliev, Higher spin gauge interactions for matter fields in two-dimensions, Phys. Lett. B 363 (1995) 51 [hep-th/9511063] [INSPIRE].

[133] K.B. Alkalaev, On higher spin extension of the Jackiw-Teitelboim gravity model, J. Phys. A 47 (2014) 365401 [arXiv: 1311.5119] [INSPIRE]. 